\title{
One-step assembly of large CRISPR arrays enables multi-functional targeting and reveals constraints on array design
}

\author{
Chunyu Liao ${ }^{1,2}$, Fani Ttofali ${ }^{1}$, Rebecca A. Slotkowski ${ }^{1}$, Steven R. Denny ${ }^{1}$, Taylor D. Cecil ${ }^{1}$, Ryan \\ T. Leenay ${ }^{1}$, Albert J. Keung ${ }^{1}$, Chase L. Beisel ${ }^{1,2}+$ \\ ${ }^{1}$ Department of Chemical and Biomolecular Engineering \\ North Carolina State University, Raleigh, NC 27695, USA \\ ${ }^{2}$ Helmholtz Institute for RNA-based Infection Research \\ Josef-Schneider-Str. 2 / D15, D-97080 Würzburg, Germany \\ tCorrespondence to chase.beisel@helmholtz-hiri.de (C.L.B.)
}

Key words: Cas9, Cpf1, C2c2, CRATES, CRISPR RNA, DNA assembly, genome editing, gene regulation

Running title: One-step assembly of CRISPR arrays 


\section{SUMMARY}

2 CRISPR-Cas systems inherently multiplex through their CRISPR arrays--whether to confer

3 immunity against multiple invaders or by mediating multi-target editing, regulation, imaging, and

4 sensing. However, arrays remain difficult to generate due to their reoccurring repeat sequences.

5 Here, we report an efficient, one-step scheme called CRATES to construct large CRISPR arrays

6 through defined assembly junctions within the trimmed portion of array spacers. We show that

7 the constructed arrays function with the single-effector nucleases Cas9, Cas12a, and Cas13a

8 for multiplexed DNA/RNA cleavage and gene regulation in cell-free systems, bacteria, and

9 yeast. We also applied CRATES to assemble composite arrays utilized by multiple Cas

10 nucleases, where these arrays enhanced DNA targeting specificity by blocking off-target sites.

11 Finally, array characterization revealed context-dependent loss of spacer activity and

12 processing of unintended guide RNAs derived from Cas12a terminal repeats. CRATES thus can

13 facilitate diverse applications requiring CRISPR multiplexing and help elucidate critical factors

14 influencing array function. 


\section{INTRODUCTION}

2 CRISPR-Cas systems represent RNA-directed immune systems whose programmable

3 nucleases have become powerful technologies for genome editing, gene regulation, imaging,

4 and diagnostics (Barrangou and Doudna, 2016; Komor et al., 2017). CRISPR technologies

5 derive from an increasing assortment of CRISPR-associated (Cas) single-effector nucleases

6 (Koonin et al., 2017; Mohanraju et al., 2016). These nucleases include the originally discovered

7 and widely-used Type II Cas9 nucleases that introduce a blunt cut into dsDNA targets

8 (Gasiunas et al., 2012; Jinek et al., 2012), the more recently discovered Type V Cas12a (or

9 Cpf1) nucleases that introduce a staggered cut into dsDNA targets and degrade ssDNA upon

10 target recognition (Chen et al., 2018; Zetsche et al., 2015), and the functionally unique Type VI

11 Cas13a (or C2c2) nucleases that cut ssRNA targets and can non-specifically degrade cellular

12 RNAs upon target recognition (Abudayyeh et al., 2016; Shmakov et al., 2015). Across this

13 diversity, CRISPR-Cas systems share an inherent capacity for multiplexing through their

14 CRISPR arrays. These arrays are composed of alternating conserved "repeats" and targeting

15 "spacers", where some prokaryotes can encode up to a few hundred spacers in a single array

16 that are stable over evolutionary timescales (Jansen et al., 2002). The transcribed array

17 undergoes processing into multiple guide RNAs (gRNAs) derived from each repeat-spacer pair.

18 Each gRNA directs the Cas nuclease to bind and cleave complementary DNA or RNA targets

19 flanked by a short protospacer-adjacent motif (PAM) or a protospacer-flanking sequence (PFS)

20 (Leenay and Beisel, 2017).

21 The multiplexing capacity of CRISPR arrays was well recognized before the advent of

22 CRISPR-Cas9 technologies (Barrangou et al., 2007; Brouns et al., 2008). However, multiplexing

23 with Cas9 outside of bacterial systems was constrained by the limited portability of RNase III

24 and the trans-activating CRISPR RNA (tracrRNA) necessary for processing of Cas9 arrays

25 (Cong et al., 2013). The invention of the single-guide RNA (sgRNA) obviated the need to co- 
1 express the tracrRNA and RNase III (Jinek et al., 2012), although the sgRNA sacrificed the

2 inherent multiplexing capability of CRISPR arrays and therefore required numerous engineering

3 workarounds to produce multiple sgRNAs (Nissim et al., 2014; Wong et al., 2016; Xie et al.,

4 2015). The discovery that the Cas 12 and Cas 13 nucleases processed CRISPR arrays through

5 their own endonucleolytic domains (East-Seletsky et al., 2016; Fonfara et al., 2016; Zetsche et

6 al., 2015; Zhong et al., 2017) intensified the pursuit of synthetic CRISPR arrays for multiplexing

7 applications. More recent examples include multiplexed genome editing and gene activation

8 with Cas12a (Tak et al., 2017; Zetsche et al., 2016), and multiplexed gene silencing with

9 Cas13a (Abudayyeh et al., 2017). Beyond these recent demonstrations, use of arrays could

10 also advance many other CRISPR technologies that have yet to employ CRISPR arrays for

11 multiplexing, such as paired nickases or Fokl fusions (Guilinger et al., 2014; Tsai et al., 2014),

12 enhanced gene drives (Noble et al., 2017), proximal CRISPR targeting (Chen et al., 2017a),

13 multi-pathogen antimicrobials (Fagen et al., 2017), multiplexed base editing (Banno et al.,

14 2018), combinatorial nucleic-acid sensing with Cas12a and Cas13a (Chen et al., 2018;

15 Gootenberg et al., 2017), and combinatorial screens (Billon et al., 2017; Kuscu et al., 2017;

16 Peters et al., 2016). However, one of the major barriers impeding multiplexing applications is

17 how to generate CRISPR arrays.

18 As a default, CRISPR arrays would be chemically synthesized as linear dsDNA by 19 commercial vendors. Unfortunately, the reoccuring repeat sequences inherent to these arrays

20 currently pose major technical complications when assembling individually synthesized

21 oligonucleotides, resulting in vendors regularly rejecting customer requests even for a minimal

22 single-spacer array. Gene synthesis has offered a more reliable means of obtaining custom

23 CRISPR arrays. However, synthesis often comes at large cost ( $\sim 5 x$ the price of a linear dsDNA)

24 and timeframes ( $\sim 1$ month), and the synthesis can often fail. As an alternative, a few groups

25 have developed different assembly methods based on annealing shorter oligonucleotides into 
repeat-spacer subunits that can be assembled sequentially or simultaneously into arrays (Table S1) (Cress et al., 2015; Gomaa et al., 2014; Tak et al., 2017; Vercoe et al., 2013; Zetsche et al.,

3 2016). For instance, one study sequentially inserted individual repeat-spacer subunits into a

4 non-target spacer to generate Cas9 arrays with up to a three spacers (Cress et al., 2015) while

5 another assembled Cas12a arrays with up to three spacers in one step by creating 5' overhangs

6 that fall within different parts of the conserved repeat (Tak et al., 2017). While these approaches

7 were used to successfully generate CRISPR arrays harboring 2 - 4 spacers, they cannot scale

8 to larger arrays and often exhibited low cloning efficiencies even for these small arrays (Table

9 S1). Therefore, the ability to easily, cheaply, and quickly generate CRISPR arrays remains an

10 impediment to the widespread use of CRISPR multiplexing and the fundamental study of array

11 processing and function.

12 Here, we present an assembly scheme for the efficient, one-step generation of large

13 CRISPR arrays. The method, which we have named CRATES ( $\underline{\text { RRISPR }}$ Assembly through

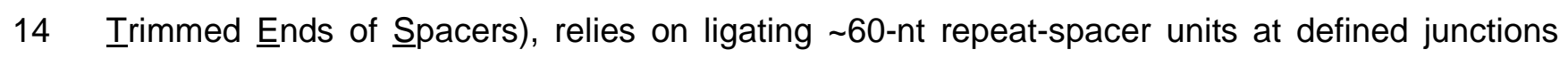

15 within the trimmed and therefore expendable portion of each spacer. The junctions allowed for

16 the efficient assembly of arrays with up to seven spacers. Using the resulting arrays, we

17 demonstrated multiplexed nucleic-acid cleavage and gene regulation with the single-effector

18 nucleases Cas9, Cas12a, and Cas13a in the bacterium E. coli, the eukaryote Saccharomyces

19 cerevisiae, and a cell-free transcription-translation (TXTL) system. Moreover, we created

20 composite arrays that were utilized by more than one nuclease, where these arrays were used

21 to demonstrate enhanced targeting specificity through the coordinated cleavage of an on-target

22 site by Cas9 and blocking of multiple off-target sites by a catalytically-dead Cas $12 a$. Finally,

23 interrogation of the arrays revealed design considerations and challenges, such as widely

24 ranging abundances of the processed gRNAs that could render an otherwise functional spacer

25 inactive and Cas12a deriving unintended gRNAs from the terminal repeat. In total, the assembly 
1 scheme is expected to streamline multiplexing with numerous CRISPR single-effector

2 nucleases and facilitate the interrogation of the processing, function, and evolution of CRISPR

3 arrays across the prokaryotic world.

5 RESULTS

6 CRATES: a one-step assembly scheme for Class 2 CRISPR arrays. Given the growing

7 interest in multiplexing with Cas12a for genome editing and gene regulation, we first sought to

8 develop a one-step assembly scheme for these arrays that relied on short oligonucleotides and

9 could scale to large arrays. Modular assembly techniques have proven effective for the one-pot

10 assembly of multiple DNA fragments (Casini et al., 2015). These techniques involve the

11 digestion of DNA fragments with Type IIS restriction enzymes, resulting in 4-nt overhangs that

12 are annealed to form the junctions between fragments in the final construct. While the

13 overhangs theoretically can be any sequence, defined sets of overhang sequences are

14 commonly used to prevent the formation of unintended junctions (e.g. between two overhangs

15 that can partially anneal) ( $\mathrm{Ng}$ and Sarkar, 2012). The question was where to insert these

16 junctions within a Cas $12 \mathrm{a}$ array without disrupting array processing or CRISPR function. The

17 original characterization of Cas12a revealed that each spacer was trimmed at its 3' end from 30

18 nts in the transcribed array to 23 nts in the processed gRNA (Fig. 1A) (Zetsche et al., 2015).

19 Furthermore, recent crystal structures of the Cas12a:gRNA ribonucleoprotein complex bound to

20 target DNA showed that only the first 20 nts of the guide portion of the gRNA participated in

21 base pairing (Swarts et al., 2017; Yamano et al., 2017). We therefore chose the trimmed region

22 of the spacer as the site of the defined junction, because spacers can accommodate virtually

23 any sequence and the junction would not be expected to participate in target recognition.

24 We next created a base construct for the assembly and expression of Cas12a arrays in

25 the bacterium Escherichia coli (Fig. 1B). The construct contained a total of six components: a 
constitutive promoter to drive transcription of the array, two Type IIS restriction sites for inserting multiple repeat-spacer subunits, a GFP reporter construct that is excised as part of array assembly, a 3' repeat so the final array begins and ends with repeats, and a terminator to halt transcription. The repeat-spacer subunits were formed by annealing two oligonucleotides of $\sim 66$ nts, thereby allowing us to readily generate 5' or 3' overhangs of any sequence. By alternating 5' and 3' overhangs and choosing sets of compatible junctions previously validated for efficient modular assembly ( $\mathrm{Ng}$ and Sarkar, 2012), we could greatly reduce the frequency of array misassembly. We chose 4-nt overhangs given their use by many modular assembly techniques,

9 although other overhang lengths could be used. The repeat-spacer subunits and the base

10 construct could then be assembled in a one-pot reaction that cycles between digestion of the 11 backbone with a Type IIS restriction enzyme and ligation by T4 DNA ligase. Figure 1B depicts

12 the assembly of a three-spacer array with four distinct junctions (5', A, B, 3'). We term the

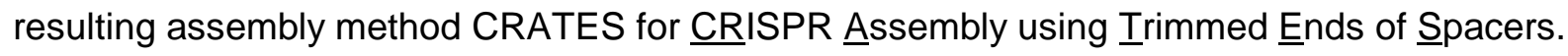

CRATES enabled efficient array assembly and revealed constraints on gRNA production.

We first explored how well CRATES could assembly arrays containing different numbers of spacers. We used the 36-nt repeats for the Cas12a nuclease from Francisella novicida U112 (FnCas12a), one of the best-characterized Cas12a nucleases to-date (Zetsche et al., 2015), and we designed up to seven spacers containing distinct 26-nt sequences and 4-nt junctions.

20 Following the assembly reaction and transformation into $E$. coli, we counted the relative number

21 of fluorescent or non-fluorescent cells, where non-fluorescent cells lost the GFP expression 22 construct and therefore would be expected to contain assembled arrays (Fig. 1C). We found 23 that the total number of transformants decreased for arrays with more spacers, although non-

24 fluorescent colonies always outnumbered fluorescent colonies by a factor of 30 up to 1,000 . In 25 contrast, only fluorescent colonies were observed in the absence of any added repeat-spacer 
1 subunits. We further performed colony PCR to assess the extent to which non-fluorescent

2 colonies harbored the expected band size (Fig. 1C). Testing five random, non-fluorescent

3 colonies yielded the correct band size $100 \%$ of the time $(5 / 5)$ for arrays with up to five spacers

4 and $60 \%$ of the time $(3 / 5)$ for arrays with seven spacers. Sanger sequencing of individual

5 colonies confirmed that the arrays contained the expected sequence. CRATES therefore

6 represents a simple and efficient way to assemble large CRISPR arrays up to and potentially

7 exceeding seven spacers.

$8 \quad$ We next asked how the seven-spacer array--the largest array we assembled--undergoes

9 processing by $\mathrm{FnCas} 12 \mathrm{a}$ when transcribed. We used a recently-developed all-E. coli cell-free

10 TXTL system to co-express the array along with FnCas12a and then performed RNA-seq

11 analysis on the purified small RNAs (Marshall et al., 2018). Sequencing showed that the

12 transcribed array was processed into 44-nt gRNAs similar to prior work (Fonfara et al., 2016;

13 Zetsche et al., 2015), resulting in loss of the 4 nt junctions in most of the sequenced gRNAs. We

14 also observed that the abundance of each gRNA varied widely, potentially impacting the ability

15 of these gRNAs to direct FnCas12a-mediated DNA targeting. Surprisingly, we also observed

16 that the terminal 3' repeat gave rise to an abundant yet unintended gRNA. Aside from the

17 potential technological complications of creating an errant gRNA, this phenomenon could lead

18 to unintended targeting by natural CRISPR-Cas systems. As a result, natural terminal repeats in

19 Cas 12a arrays may have been under selective pressure to accumulate mutations that prevent

20 processing, helping explain the high frequency of mutations in terminal repeats but no other

21 repeats. Correspondingly, analysis of diverse terminal repeats from 14 Type V-A CRISPR-Cas

22 systems revealed that $79 \%(11 / 14)$ of the native terminal repeats harbored mutations known to

23 disrupt Cas12a recognition (Fig. S1) (Fonfara et al., 2016; Zetsche et al., 2015). It remains to be

24 seen whether the terminal repeat for the other three CRISPR-Cas systems gives rise to a 
1 functional gRNA. These insights aside, we conclude that the generated Cas12a array can be

2 transcribed and undergo processing to form multiple gRNAs.

4 CRATES revealed context-dependent loss of FnCas12a spacer activity. We next

5 investigated the targeting activity of the assembled arrays based on plasmid clearance in E. coli

6 (Fig. 2A). As part of the assay, a targeting or no-spacer CRISPR array plasmid was

7 transformed into cells harboring the FnCas12a plasmid and a plasmid containing the target

8 sequence. Successful plasmid clearance resulted in a large reduction in the number of

9 antibiotic-resistant colonies for the targeting array versus the no-spacer array. We designed

10 three spacers against three distinct target sequences: the lacZ promoter (blue), a 5' portion of

11 the gfp coding region (purple), and a 3' portion of the gfp coding region (green). As expected,

12 the CRISPR plasmid with each single spacer cleared only its cognate target plasmid (Fig. 2A).

13 The clearance activity was similar in the presence or absence of the junction sequence across

14 all three spacers (Fig. S2), ruling out an obvious effect of the junction. We then generated all six

15 configurations of the three-spacer arrays (Fig. 2A). Five out of the six arrays cleared all three

16 plasmids. However, one array $(\mathrm{PcF}-2 / 3 / 1)$ was unable to clear the plasmid with the lacZ

17 promoter sequence (blue). Given that the associated spacer drove robust plasmid clearance in

18 the same position in another array (PcF-3/2/1), we attribute the lost activity to the specific

19 context of the spacer in the array.

20 RNA-seq analysis of the seven-spacer array revealed widely varying abundances of the

21 processed gRNAs, where a low-abundance gRNA may explain the observed lack of plasmid

22 clearance activity. To evaluate this possibility directly, we performed RNA-seq analysis on the

$23 \mathrm{PcF}-2 / 3 / 1$ and PcF-1/3/2 arrays that negligibly or efficiently cleared the plasmid with the lacZ

24 promoter sequence, respectively (Fig. 2B). The lacZ promoter-targeting gRNA in PcF-2/3/1 had

25 the lowest abundance across the two arrays and was $~ 4$-fold lower than the same gRNA in 
1 PcF-1/3/2 relative to the middle spacer in each array--all in line with poor plasmid clearance

2 activity. We also noticed that the most-abundant gRNAs were derived from the terminal repeat.

3 These gRNAs could be titrating out available FnCas12a protein, resulting in negligible DNA

4 targeting by less-abundant gRNAs. Given that the terminal repeat is not necessary to generate

5 a functional gRNA (Zetsche et al., 2015), we removed this repeat from both arrays and repeated

6 the plasmid-clearance assay. However, removing the terminal repeat did not appreciably

7 improve plasmid clearance (Fig. S3A). Therefore, other factors likely account for the context-

8 dependent loss of targeting activity.

$9 \quad$ We also explored whether array assembly and multiplexed plasmid clearance could 10 extend to other Cas12a proteins. We chose the Cas12a nuclease from Acidaminococcus sp.

11 BV3L6, one of the most commonly used variants of Cas12a (Tang et al., 2017; Zetsche et al.,

12 2015). As expected, we were able to readily generate three-spacer arrays and achieve

13 multiplexed plasmid clearance. All three spacers led to robust plasmid clearance whether in

14 one-spacer arrays or in a three-spacer array (Fig. S3B), confirming that CRATES can be 15 extended to different Cas12a nucleases.

17 CRATES facilitated multiplexed gene regulation by dCas12a. Following our demonstration

18 of multiplexed DNA cleavage with Cas12a, we next explored the use of CRATES to enact 19 multiplexed gene regulation. Prior work identified three RuvC endonucleolytic domains within 20 FnCas12a, where single point mutations completely (D917A, E1006A) or partially (D1255A) 21 disrupted DNA cleavage activity while retaining DNA binding activity in vitro (Fonfara et al., 22 2016; Zetsche et al., 2015). Single or double point mutations of these domains have been used 23 for programmable gene regulation with Cas12a in bacteria, mammalian cells, and plants 24 (Leenay et al., 2016; Tak et al., 2017; Tang et al., 2017), yet the mutations remain to be 25 compared systematically. We therefore generated variants of Cas12a containing single, double, 
1 or triple mutations and then measured DNA targeting of each catalytically-dead variant of

2 FnCas12a (dFnCas12a) based on plasmid clearance or gene repression in E. coli (Fig. 3A). For

3 either assay, each variant was co-expressed with a single-spacer array targeting the lacZ

4 promoter controlling gfp on a transformed plasmid. The transformations were performed in an $E$.

5 coli strain lacking this promoter to prevent incidental genome targeting. We found that all tested

6 mutations eliminated any measurable plasmid clearance activity and yielded GFP repression by

7 flow cytometry analysis. Interestingly, one of the single mutations (E1006A) exhibited greatly

8 reduced repression activity that was reversed with additional mutations. Aside from the E1006A

9 single mutation, all other mutations yielded similar strengths of gene repression.

10 We selected the triple mutant (D917A, E1006A, D1255A) to evaluate multiplexed gene

11 repression in E. coli. We designed spacers to target the lacZ, laclq, or araB promoter controlling

12 gfp in separate plasmids. We then constructed single-spacer or three-spacer arrays and

13 performed flow cytometry analysis to determine the extent of gene repression for each

14 combination of promoter-arrays in comparison to the no-spacer array (Fig. 3B). As expected,

15 each single-spacer array silenced GFP expression only from the cognate promoter, whereas all

16 three-spacer arrays that we tested silenced GFP for all promoters. We also observed some

17 variability in GFP silencing between the tested three-spacer arrays, suggesting some impact of

18 the spacer context, although there was no complete loss of silencing activity.

19 One of the promising uses of Cas12a is multiplexed gene activation in eukaryotic cells,

20 yet only one study has demonstrated this capability to-date (Tak et al., 2017). We therefore

21 sought to evaluate gene activation in the budding yeast Saccharomyces cerevisiae using a

22 fusion between the FnCas12a double mutant (D917A, E1006A) and the transcriptional activator

23 domain VP64. For these experiments, we constructed a new base CRISPR plasmid with the

24 SNR52 snoRNA promoter and a polyT terminator. We then constructed single-spacer or three-

25 spacer arrays targeting different locations around the CYC1 promoter, which drives expression 
1 of a downstream chromosomal copy of yeGFP. Flow cytometry analysis showed that each

2 single-spacer array yielded up to $6.6 \%$ yEGFP-expressing cells, while the three-spacer arrays

3 consistently yielded 11\% yEGFP-expressing cells (Figs. 3C, S4). Furthermore, the average

4 fluorescence values were higher for cells with the three-spacer arrays than any of the single-

5 spacer arrays (Fig. S4), mirroring the synergistic impact of recruiting multiple activators to the

6 same promoter as observed for dCas9 (Maeder et al., 2013; Perez-Pinera et al., 2013). The

7 observed activation of yEGFP expression was lost when using dFnCas12a without the fused

8 transcriptional activator domain (Fig. S4). In total, we showed that CRATES can be used to

9 generate Cas12a arrays for multiplexed gene repression in $E$. coli and multiplexed gene

10 activation in yeast, creating the potential of implementing these arrays in different eukaryotic

11 organisms.

13 CRATES is compatible with other Class 2 CRISPR nucleases. We have so far shown that

14 CRATES can be used to construct CRISPR arrays utilized by Cas12a. However, the basis of

15 the assembly method--junctions in the trimmed portion of the spacers--is not limited to Cas $12 \mathrm{a}$

16 arrays. Instead, all other Class 2 CRISPR-Cas systems that have been characterized to-date

17 exhibit spacer trimming (Deltcheva et al., 2011; East-Seletsky et al., 2016; Shmakov et al.,

18 2015). This common feature would suggest that CRATES could be compatible with any Class 2

19 CRISPR nuclease. As a start, we adapted CRATES to assemble CRISPR arrays recognized by

20 the Type II-A single-effector nuclease Cas9 from Streptococcus pyogenes (SpCas9), arguably

21 the most widely used CRISPR nuclease to-date (Fig. 4A). The major alteration to the base

22 plasmid was placing the repeat upstream of the 5' restriction site so the assembly junction

23 would fall within $5^{\prime}$ trimmed region of each spacer. Co-expressing the resulting arrays, Cas9,

24 and the tracrRNA in a bacterium with RNase III would lead to processing of the CRISPR array

25 into individual gRNAs (Deltcheva et al., 2011). To demonstrate this functionality, we designed 
1 spacers targeting three plasmids respectively. We then cloned three single-spacer arrays and

2 one three-spacer array and performed the plasmid clearance assays in E. coli. As expected, the

3 single-spacer arrays only cleared their cognate target plasmid, while the three-spacer assay

4 cleared all three plasmids.

$5 \quad$ As a further demonstration, we used CRATES to explore multiplexed RNA sensing with

6 the Type VI single-effector nuclease Cas13a from Leptotrichia shahii (LsCas13a). This nuclease

7 possesses the unique ability to target RNA, where target recognition activates a separate

8 endonuclease domain within LsCas13a that non-specifically degrades cellular RNAs

9 (Abudayyeh et al., 2016). The non-specific activity was recently exploited for the sensitive

10 detection of viral RNAs (Gootenberg et al., 2017, 2018), while this same activity was sufficiently

11 low in human cells to allow programmable and targeted gene silencing with the wild-type

12 nuclease (Abudayyeh et al., 2017). Because the LsCas13a spacer is trimmed on the 3' end as

13 part of gRNA processing (East-Seletsky et al., 2016; Shmakov et al., 2015), we developed a

14 base assembly construct similar to that for Cas12a (Fig. 4C). We then devised an RNA-sensing

15 assay using TXTL, where DNA encoding LsCas13a and the constructed CRISPR array were

16 mixed with DNA expressing a target RNA and a non-targeted GFP reporter (Fig. S5A,B). The

17 GFP transcript would undergo non-specific degradation only when the processed gRNA is

18 paired with its target, resulting in a reduction of GFP fluorescence. We created three unique

19 spacers and three complementary targets placed downstream of a constitutive promoter. As

20 expected, GFP expression was reduced compared to the no-spacer array only when the gRNA

21 and its target were both present. Furthermore, the three-spacer arrays reduced GFP in the

22 presence of any of the three target transcripts but not of a non-targeted transcript, resulting in

23 the simultaneous sensing of multiple RNA species (Figs. 4D, S5C). Interestingly, some

24 transcripts led to more potent GFP silencing than others, presumably due to ranging secondary 
1 structures impacting the accessibility of the target sequence as reported previously (Abudayyeh

2 et al., 2016).

4 CRATES-assembled composite arrays mediated coordinated DNA targeting by multiple

5 Cas nucleases. Emerging examples have shown that orthogonal Cas nucleases can be

6 combined to simultaneously perform multiple CRISPR functions, such as conferring immune

7 defense and gene regulation (Esvelt et al., 2013), improving DNA accessibility through binding a

8 proximal site (Chen et al., 2017a), or combining gene disruption and activation in a genome-

9 wide screen (Najm et al., 2018). However, in each case, the gRNAs had to be transcribed from

10 separate expression constructs. We therefore asked if CRATES could be used to generate

11 CRISPR arrays that are processed into gRNAs recognized by multiple Cas nucleases--in what

12 we term "composite" arrays. The simplest arrangement for a composite array would be multiple

13 CRISPR arrays inserted sequentially into the same transcript. Figure 5A illustrates a composite

14 array composed of a two-spacer SpCas9 array followed by a two-spacer FnCas12a array (Fig.

15 5B).

16 To explore the technological potential of composite arrays, we first assessed the ability

17 of these arrays to coordinate plasmid clearance by SpCas 9 and gene repression by the

18 FnCas12a triple mutant (D917A, E1006A, D1255A) in E. coli (Fig. 5C). As part of the

19 assessment, we designed two spacers for each nuclease targeting the lacZ or laclq promoter

20 controlling gfp in the reporter plasmid. We then constructed arrays containing each spacer or all

21 four spacers in two configurations (Fig. 5C). E. coli cells harboring the SpCas9 or FnCas12a

22 triple-mutant plasmid and either GFP reporter plasmid were transformed with each CRISPR

23 plasmid. We then assessed the transformation efficiency (SpCas9) or GFP fluorescence

24 (dFnCas12a) in comparison to a no-spacer array. As expected, the single spacers yielded

25 plasmid clearance or GFP repression when matched with their nuclease and targeted plasmid, 
1 while one of the four-spacer composite arrays (Pc7/8/9/10) yielded plasmid clearance or GFP

2 repression comparable to that of all single-spacer arrays. Interestingly, the other four-spacer

3 composite array with swapped SpCas9 spacers (Pc8/7/9/10) exhibited greatly reduced plasmid

4 clearance of one target plasmid by SpCas9, paralleling the diminished activity of one of the

5 FnCas12a spacers used for plasmid clearance (Fig. 2A).

6

7 Composite arrays can improve on-target specificity through coordinated blocking of off-

8 target sites. Following the proof-of-principle demonstration of composite arrays, we sought a

9 more practical application that benefits from coordinated DNA binding and cleavage. One

10 unexplored application is reducing off-target activity by using a catalytically-dead nuclease to

11 bind--and therefore block--known off-target sites. This strategy could be used in combination

12 with existing approaches for diminishing off-target editing such as modified gRNAs or improved-

13 specificity variants of Cas9 (Chen et al., 2017b; Fu et al., 2014; Hu et al., 2018; Kleinstiver et al.,

14 2016a; Slaymaker et al., 2016; Yin et al., 2018), and it could directly exploit a growing suite of

15 experimental techniques for the unbiased detection of off-target sites (Tsai and Keith Joung,

16 2016). Most importantly, it could allow the selection of target sites that otherwise might have

17 been rejected because of known off-target locations.

18 We chose SpyCas9 for on-target cleavage and the FnCas12a triple mutant to block off-

19 target sites (Fig. 5C). We further selected one of the first examples of off-target cleavage by

20 SpCas9 in human cells: an on-target site in WAS CR-4 (P-on), and two off-target sites in STK25

21 (P-off1) and GNHR2 (P-off2) (Fig. S6A) (Fu et al., 2013). The target sites for FnCas12a were

22 chosen so the R-loop would extend through the NGG PAM recognized by SpCas9, thereby

23 presumably preventing any DNA recognition.

24 We used TXTL as a rapid and dynamic means to assess whether the catalytically-dead

25 FnCas12a could block SpCas9-mediated cleavage of the off-target sites but not the on-target 
1 site. As part of the assay, the target sites were inserted $\sim 170$ bps upstream of the P70a

2 promoter controlling gfp. Cleavage by SpCas9 would result in rapid degradation by RecBCD

3 and loss of GFP production (Fig. S6B) (Maxwell et al., 2018). Using CRATES, we assembled a

4 variant of the composite array encoding the targeting SpCas9 sgRNA upstream of a two-spacer

5 FnCas12a array, where both configurations of the FnCas12a spacers (Psg/b1/b2 and

$6 \mathrm{Psg} / \mathrm{b} 2 / \mathrm{b} 1)$ were tested. We then assayed the cleavage activity of SpCas9 on each site by first

7 adding to the TXTL reaction the FnCas12a triple-mutant plasmid, a reporter plasmid, and either

8 the plasmid encoding a composite array or two plasmids separately encoding the sgRNA and a

9 single-spacer FnCas12a array. We then added the plasmid encoding SpCas9 and measured

10 GFP fluorescence over time. The protection efficiency was then calculated based on the rate of

11 GFP production in comparison to that when expressing a no-spacer FnCas12a array and the

12 targeting sgRNA (0\% protection) or a non-targeting sgRNA (100\% protection). We found that

13 both tested composite arrays inhibited cleavage by SpCas9 at both off-target sites at

14 efficiencies similar to those when expressing the SpCas9 sgRNA and single-spacer FnCas12a

15 arrays separately (Fig. 5E). Critically, there was negligible protection for the on-target site.

16 These results therefore offer an proof-of-principle demonstration of using composite arrays to

17 improve on-target specificity by Cas nucleases.

\section{DISCUSSION}

20 We have devised and validated a technique we term CRATES for the efficient, one-step

21 generation of large CRISPR arrays. The technique relies on assembling multiple repeat-spacer

22 subunits using defined junction sequences within the trimmed portion of the CRISPR spacers.

23 By specifying the sequence and 5' or 3' directionality of the overhang that forms the junction, we

24 could minimize unintended pairing between non-adjacent repeat-spacer subunits. We showed

25 that the technique could generate CRISPR arrays harboring up to seven spacers with high 
1 efficiency. While the technique may extend to even larger arrays, the cloning efficiency could be

2 further improved by using different junction lengths (e.g. between 1 and 10 nts for Cas9

3 spacers) and by subjecting the repeat-spacer oligonucleotides to further purification to remove

4 truncated products.

$5 \quad$ We showed that the assembled arrays could be utilized by three different single-effector 6 nucleases (Cas9, Cas12a, Cas13a) that yielded multiplexed DNA or RNA targeting in bacteria, 7 eukaryotic cells, and cell-free systems. Given the numerous applications for CRISPR

8 technologies that could benefit from multiplexing--from genome editing, epigenetic regulation,

9 and gene drives to nucleic-acid sensing, antimicrobials, and genetic screens--CRATES has the

10 potential to be widely implemented. Two particularly noteworthy uses are for gene drives and

11 genetic screens. Gene drives utilize a CRISPR nuclease transferring itself and any adjacent

12 genetic cargo to the matching locus in a sister chromosome, thereby allowing the rapid spread

13 of the cargo through a wild population (Esvelt et al., 2014). Because this technique relies on

14 homologous recombination, indel formation through non-homologous end joining (NHEJ) would

15 prevent further editing and create a drive-immune member of the population. By targeting

16 multiple sites in the matching locus through a generated array, disruption of the gene drive

17 through indel formation could be greatly reduced or even eliminated due to the vanishingly small

18 probability that all sites undergo repair through NHEJ simultaneously. In the second example,

19 CRISPR technologies have been used for genome-wide screens based on gene disruption,

20 activation, or repression (Shalem et al., 2015). While these screens principally relied on a library

21 of single sgRNAs, there are recent examples where libraries containing sgRNA pairs were used

22 in human cells to identify synthetic-lethal genes as well as gene pairs that drive cancer

23 proliferation or interact with tumor protein p53 (Najm et al., 2018; Wong et al., 2016). In both

24 examples, the sgRNAs had to be cloned sequentially. CRATES therefore could build on these

25 approaches through the one-step assembly of array libraries that extend beyond two targets, 
1 allowing the combinatorial screening of large and potentially redundant factors such as virulence

2 factors, small RNAs, or two-component systems.

We also assembled composite arrays that could be utilized by multiple nucleases (Fig.

4 5). These arrays created the opportunity to enact multiple multiplexed functions by CRISPR

5 nucleases from a single transcript. While expressing or delivering more than one nuclease can

6 be cumbersome, there have been a few applications that benefited from multiple nucleases. In

7 one example, the frequency of gene editing was enhanced by directing catalytically-dead

8 SpCas9 to bind proximally to the site targeted by an orthogonal and catalytically active Cas 9 or

9 Cas12a (Chen et al., 2017a). In another example cited above, the SpCas9 and Staphylococcus

10 aureus (Sa)Cas9 were used to perform combinatorial screens, where SpCas9 created indels

11 while SaCas9 upregulated gene expression (Najm et al., 2018). In both examples, composite

12 arrays could be used to expand the number of targeting gRNAs produced at one time--whether

13 to enhance editing at one or multiple sites or to increase the number of target genes in a

14 combinatorial screen. We also reported another use of composite arrays based on blocking

15 known off-target sites (Fig. 5D). While this approach remains to be demonstrated in cells, it

16 suggests another means to reduce off-target effects that would complement existing strategies

17 and resurrect the use of targets with known off-target sites. This feature would be especially

18 important for edits that can only be achieved through a limited number of target sites, such as

19 for reversing single-nucleotide polymorphisms associated with human disease or performing

20 site-specific integrations (Bulik-Sullivan et al., 2015; Eyquem et al., 2017).

21 Our assembly scheme depended on the insertion of junctions into the trimmed portions

22 of spacers within a CRISPR array. To-date, spacer trimming has been associated with all Class

232 CRISPR-Cas systems (II, V, VI) and with some Class 1 Type III CRISPR-Cas systems (Hale

24 et al., 2009). On the other hand, Type I CRISPR-Cas systems have not been reported to

25 undergo spacer trimming because of the mechanism of ribonucleoprotein complex assembly 
1 (Brouns et al., 2008) and because at least one system has been shown to reject mismatches at

2 the PAM-distal end of the spacer (Szczelkun et al., 2014). CRATES could generate CRISPR

3 arrays for Type I systems by using part of the natural spacer sequence as the junction, where

4 the use of 5' or 3' overhangs could be optimized to help eliminate potential cross-interactions

5 between non-adjacent repeat-spacer subunits. This potential limitation aside, Class 2 CRISPR-

6 Cas systems and their single-effector nucleases remain the primary source of CRISPR

7 technologies and would benefit directly from CRATES.

8 By testing a large cohort of assembled CRISPR arrays, we observed two unique

9 features that could impact their technological potential. One unique feature was spacers being

10 inactive in multi-spacer arrays despite being fully active in single-spacer arrays. In particular, we

11 found an FnCas12a spacer and an SpCas9 spacer that lost their ability to elicit targeted plasmid

12 clearance in the respective context of a three-spacer array (Fig. 2A) or a four-spacer composite

13 array (Fig. 5C). The effect could not be solely explained by the location of the spacer, as

14 swapping the order of the other spacers restored activity. Instead, the lost activity appears to be

15 context-dependent and could involve interactions with adjacent spacers such as through

16 secondary structure formation. While the lost activity was sparingly observed in our work

17 (individual spacers in 2/26 tested arrays) and was not observed in the few instances in which

18 individual spacers in synthetic arrays were assayed (Luo et al., 2016; Zetsche et al., 2016), it

19 nonetheless could represent an important issue particularly for arrays that cannot be fully

20 validated before they are implemented (e.g. combinatorial screens, editing in multicellular

21 organisms).

Although the exact mechanism underlying the loss of spacer activity remains unclear, we

23 provided evidence linking spacer activity to the low abundance of the resulting gRNA (Fig. 2B).

24 Interestingly, natural CRISPR arrays exhibit widely varying abundances of the processed

25 gRNAs that is only partially explained by the proximity to the transcriptional start site (Carte et 
1 al., 2014; Deltcheva et al., 2011; Plagens et al., 2014; Zetsche et al., 2015). Instead, gRNA

2 abundance varied widely and was not limited to any single type of CRISPR-Cas system, 3 suggesting that this is a common phenomenon impacting the processing of natural and

4 synthetic gRNAs. More experiments will be necessary to determine why some gRNAs are lower

5 in abundance than others, the connection between abundance and activity, and whether design

6 rules can be elucidated to ensure consistent and high gRNA abundance even for large CRISPR

7 arrays.

8 The second unique feature that we observed was FnCas12a deriving a gRNA from the

9 terminal repeat in a CRISPR array. This observation was unexpected given that only spacers

10 flanked by two repeats would be expected to become gRNAs. The more concerning ramification

11 is that the terminal repeat-derived gRNA could lead to unintended targeting. From a natural

12 perspective, we provided evidence that terminal repeats for Cas $12 \mathrm{a}$ arrays have been under

13 negative selection to prevent formation of a gRNA, resulting in mutations within the terminal

14 repeat that disrupt processing. Correspondingly, the one native Cas 12a array subjected to RNA-

15 seq analysis--from FnCas12a--did not show any gRNAs derived from the terminal repeat

16 (Zetsche et al., 2015). From a technological perspective, Cas12a gRNAs can be functionally

17 expressed without a 3' repeat (Kleinstiver et al., 2016b; Zetsche et al., 2015); however, the

18 terminal repeat was important when deriving gRNAs from eukaryotic mRNAs (Zhong et al.,

19 2017). Our results suggest that naturally occurring terminal repeats could be readily used to 20 prevent the generation of an errant gRNA while preserving proper processing and activity of 21 upstream gRNAs.

\section{ACKNOWLEDGEMENTS}

24 We thank Jörg Vogel and Lars Barquist for critical comments. The pCas9 plasmid was a gift 25 from Luciano Marraffini (Addgene plasmid \# 42876). The work was supported through funding 
from the NIH (1R35GM119561 to C.L.B. and 1DP1DA044359 to A.J.K.), the North Carolina State University Summer Undergraduate Research Grant (to T.D.C.), Agilent Technologies (Gift \#3926 to C.L.B.), and the Camille \& Henry Dreyfus Foundation (2017-137 to C.L.B.).

\section{DECLARATION OF INTERESTS}

6 C.L.B. is a co-founder and scientific advisory board member of Locus Biosciences and has

7 submitted provisional patent applications on CRISPR technologies.

\section{$9 \quad$ AUTHOR CONTRIBUTIONS}

10 C.L., and R.T.L., and C.L.B. conceived this study. C.L. and C.L.B. designed the experiments.

11 C.L., F.T., R.A.S., and S.R.D. performed the array cloning and experiments with bacteria and

12 TXTL; C.L. and C.L.B. analyzed the data. A.J.K. and T.D.C. conducted the experiments with

13 yeast. R.T.L. analyzed the RNA sequencing data. C.L. and C.L.B. wrote the manuscript, which

14 was read and approved by all authors.

\section{REFERENCES}

17 Abudayyeh, O.O., Gootenberg, J.S., Konermann, S., Joung, J., Slaymaker, I.M., Cox, D.B.T.,

18 Shmakov, S., Makarova, K.S., Semenova, E., Minakhin, L., et al. (2016). C2c2 is a single-

19 component programmable RNA-guided RNA-targeting CRISPR effector. Science 353, aaf5573.

20 Abudayyeh, O.O., Gootenberg, J.S., Essletzbichler, P., Han, S., Joung, J., Belanto, J.J.,

21 Verdine, V., Cox, D.B.T., Kellner, M.J., Regev, A., et al. (2017). RNA targeting with CRISPR-

22 Cas13. Nature 550, 280-284.

23 Afroz, T., Biliouris, K., Kaznessis, Y., and Beisel, C.L. (2014). Bacterial sugar utilization gives 24 rise to distinct single-cell behaviours. Mol. Microbiol. 93, 1093-1103.

25 Banno, S., Nishida, K., Arazoe, T., Mitsunobu, H., and Kondo, A. (2018). Deaminase-mediated

26 multiplex genome editing in Escherichia coli. Nat Microbiol 3, 423-429.

27 Barrangou, R., and Doudna, J.A. (2016). Applications of CRISPR technologies in research and 28 beyond. Nat. Biotechnol. 34, 933-941. 
Barrangou, R., Fremaux, C., Deveau, H., Richards, M., Boyaval, P., Moineau, S., Romero, D.A., and Horvath, P. (2007). CRISPR provides acquired resistance against viruses in prokaryotes. Science 315, 1709-1712.

Billon, P., Bryant, E.E., Joseph, S.A., Nambiar, T.S., Hayward, S.B., Rothstein, R., and Ciccia, A. (2017). CRISPR-mediated base editing enables efficient disruption of eukaryotic genes through induction of STOP codons. Mol. Cell 67, 1068-1079.e4.

Brouns, S.J.J., Jore, M.M., Lundgren, M., Westra, E.R., Slijkhuis, R.J.H., Snijders, A.P.L., Dickman, M.J., Makarova, K.S., Koonin, E.V., and van der Oost, J. (2008). Small CRISPR RNAs guide antiviral defense in prokaryotes. Science 321, 960-964.

Bulik-Sullivan, B., Finucane, H.K., Anttila, V., Gusev, A., Day, F.R., Loh, P.-R., ReproGen Consortium, Psychiatric Genomics Consortium, Genetic Consortium for Anorexia Nervosa of the Wellcome Trust Case Control Consortium 3, Duncan, L., et al. (2015). An atlas of genetic correlations across human diseases and traits. Nat. Genet. 47, 1236-1241.

Carte, J., Christopher, R.T., Smith, J.T., Olson, S., Barrangou, R., Moineau, S., Glover, C.V.C., 3rd, Graveley, B.R., Terns, R.M., and Terns, M.P. (2014). The three major types of CRISPRCas systems function independently in CRISPR RNA biogenesis in Streptococcus thermophilus. Mol. Microbiol. 93, 98-112.

Casini, A., Storch, M., Baldwin, G.S., and Ellis, T. (2015). Bricks and blueprints: methods and standards for DNA assembly. Nat. Rev. Mol. Cell Biol. 16, 568-576.

Chen, F., Ding, X., Feng, Y., Seebeck, T., Jiang, Y., and Davis, G.D. (2017a). Targeted activation of diverse CRISPR-Cas systems for mammalian genome editing via proximal CRISPR targeting. Nat. Commun. 8, 14958.

Chen, J.S., Dagdas, Y.S., Kleinstiver, B.P., Welch, M.M., Sousa, A.A., Harrington, L.B., Sternberg, S.H., Joung, J.K., Yildiz, A., and Doudna, J.A. (2017b). Enhanced proofreading governs CRISPR-Cas9 targeting accuracy. Nature 550, 407-410.

Chen, J.S., Ma, E., Harrington, L.B., Da Costa, M., Tian, X., Palefsky, J.M., and Doudna, J.A. (2018). CRISPR-Cas12a target binding unleashes indiscriminate single-stranded DNase activity. Science (in press).

Cong, L., Ran, F.A., Cox, D., Lin, S., Barretto, R., Habib, N., Hsu, P.D., Wu, X., Jiang, W., Marraffini, L.A., et al. (2013). Multiplex genome engineering using CRISPR/Cas systems. Science 339, 819-823.

Cress, B.F., Duhan Toparlak, Ö., Guleria, S., Lebovich, M., Stieglitz, J.T., Englaender, J.A., Andrew Jones, J., Linhardt, R.J., and Koffas, M.A.G. (2015). CRISPathBrick: modular combinatorial assembly of Type II-A CRISPR arrays for dCas9-mediated multiplex transcriptional repression in E. coli. ACS Synth. Biol. 4, 987-1000.

Deltcheva, E., Chylinski, K., Sharma, C.M., Gonzales, K., Chao, Y., Pirzada, Z.A., Eckert, M.R., Vogel, J., and Charpentier, E. (2011). CRISPR RNA maturation by trans-encoded small RNA and host factor RNase III. Nature 471, 602-607.

East-Seletsky, A., O'Connell, M.R., Knight, S.C., Burstein, D., Cate, J.H.D., Tjian, R., and 
Doudna, J.A. (2016). Two distinct RNase activities of CRISPR-C2c2 enable guide-RNA processing and RNA detection. Nature 538, 270-273.

Esvelt, K.M., Mali, P., Braff, J.L., Moosburner, M., Yaung, S.J., and Church, G.M. (2013). 1121.

Esvelt, K.M., Smidler, A.L., Catteruccia, F., and Church, G.M. (2014). Concerning RNA-guided gene drives for the alteration of wild populations. Elife 3, e03401.

Eyquem, J., Mansilla-Soto, J., Giavridis, T., van der Stegen, S.J.C., Hamieh, M., Cunanan, CRISPR/Cas9 enhances tumour rejection. Nature 543, 113-117.

11 Fagen, J.R., Collias, D., Singh, A.K., and Beisel, C.L. (2017). Advancing the design and delivery of CRISPR antimicrobials. Current Opinion in Biomedical Engineering 4, 57-64.

Fonfara, I., Richter, H., Bratovič, M., Le Rhun, A., and Charpentier, E. (2016). The CRISPRassociated DNA-cleaving enzyme Cpf1 also processes precursor CRISPR RNA. Nature 532, 517-521.

Fu, Y., Foden, J.A., Khayter, C., Maeder, M.L., Reyon, D., Joung, J.K., and Sander, J.D. (2013). High-frequency off-target mutagenesis induced by CRISPR-Cas nucleases in human cells. Nat. Biotechnol. 31, 822-826.

Fu, Y., Sander, J.D., Reyon, D., Cascio, V.M., and Joung, J.K. (2014). Improving CRISPR-Cas nuclease specificity using truncated guide RNAs. Nat. Biotechnol. 32, 279-284.

Garamella, J., Marshall, R., Rustad, M., and Noireaux, V. (2016). The all E. coli TX-TL toolbox

Gasiunas, G., Barrangou, R., Horvath, P., and Siksnys, V. (2012). Cas9-crRNA ribonucleoprotein complex mediates specific DNA cleavage for adaptive immunity in bacteria. Proc. Natl. Acad. Sci. U. S. A. 109, E2579-E2586. MBio 5, e00928-13.

Gootenberg, J.S., Abudayyeh, O.O., Lee, J.W., Essletzbichler, P., Dy, A.J., Joung, J., Verdine, Cas13a/C2c2. Science 356, 438-442.

Gootenberg, J.S., Abudayyeh, O.O., Kellner, M.J., Joung, J., Collins, J.J., and Zhang, F. (2018).

34 Science (in press).

Guilinger, J.P., Thompson, D.B., and Liu, D.R. (2014). Fusion of catalytically inactive Cas9 to 
M.P. (2009). RNA-guided RNA cleavage by a CRISPR RNA-Cas protein complex. Cell 139, 945-956.

Hu, J.H., Miller, S.M., Geurts, M.H., Tang, W., Chen, L., Sun, N., Zeina, C.M., Gao, X., Rees, H.A., Lin, Z., et al. (2018). Evolved Cas9 variants with broad PAM compatibility and high DNA specificity. Nature (in press).

Jansen, R., van Embden, J.D.A., Gaastra, W., and Schouls, L.M. (2002). Identification of a novel family of sequence repeats among prokaryotes. OMICS 6, 23-33.

Jinek, M., Chylinski, K., Fonfara, I., Hauer, M., Doudna, J.A., and Charpentier, E. (2012). A programmable dual-RNA-guided DNA endonuclease in adaptive bacterial immunity. Science 337, 816-821.

Keung, A.J., Bashor, C.J., Kiriakov, S., Collins, J.J., and Khalil, A.S. (2014). Using targeted chromatin regulators to engineer combinatorial and spatial transcriptional regulation. Cell 158, 110-120.

Khalil, A.S., Lu, T.K., Bashor, C.J., Ramirez, C.L., Pyenson, N.C., Joung, J.K., and Collins, J.J. (2012). A synthetic biology framework for programming eukaryotic transcription functions. Cell 150, 647-658.

Kleinstiver, B.P., Pattanayak, V., Prew, M.S., Tsai, S.Q., Nguyen, N.T., Zheng, Z., and Joung, J.K. (2016a). High-fidelity CRISPR-Cas9 nucleases with no detectable genome-wide off-target effects. Nature 529, 490-495.

Kleinstiver, B.P., Tsai, S.Q., Prew, M.S., Nguyen, N.T., Welch, M.M., Lopez, J.M., McCaw, Z.R., Aryee, M.J., and Joung, J.K. (2016b). Genome-wide specificities of CRISPR-Cas Cpf1 nucleases in human cells. Nat. Biotechnol. 34, 869-874.

Komor, A.C., Badran, A.H., and Liu, D.R. (2017). CRISPR-based technologies for the manipulation of eukaryotic genomes. Cell 168, 20-36.

Koonin, E.V., Makarova, K.S., and Zhang, F. (2017). Diversity, classification and evolution of CRISPR-Cas systems. Curr. Opin. Microbiol. 37, 67-78.

Kuscu, C., Parlak, M., Tufan, T., Yang, J., Szlachta, K., Wei, X., Mammadov, R., and Adli, M. (2017). CRISPR-STOP: gene silencing through base-editing-induced nonsense mutations. Nat. Methods 14, 710-712.

Leenay, R.T., and Beisel, C.L. (2017). Deciphering, communicating, and engineering the CRISPR PAM. J. Mol. Biol. 429, 177-191.

Leenay, R.T., Maksimchuk, K.R., Slotkowski, R.A., Agrawal, R.N., Gomaa, A.A., Briner, A.E., Barrangou, R., and Beisel, C.L. (2016). Identifying and visualizing functional PAM diversity across CRISPR-Cas systems. Mol. Cell 62, 137-147.

Luo, M.L., Jackson, R.N., Denny, S.R., Tokmina-Lukaszewska, M., Maksimchuk, K.R., Lin, W., Bothner, B., Wiedenheft, B., and Beisel, C.L. (2016). The CRISPR RNA-guided surveillance complex in Escherichia coli accommodates extended RNA spacers. Nucleic Acids Res. 44, 7385-7394. 
Maeder, M.L., Linder, S.J., Cascio, V.M., Fu, Y., Ho, Q.H., and Joung, J.K. (2013). CRISPR RNA-guided activation of endogenous human genes. Nat. Methods 10, 977-979.

Marshall, R., Maxwell, C.S., Collins, S.P., Jacobsen, T., Luo, M.L., Begemann, M.B., Gray, B.N., January, E., Singer, A., He, Y., et al. (2018). Rapid and scalable characterization of CRISPR technologies using an E. coli cell-free transcription-translation system. Mol. Cell 69, 146157.e3.

Maxwell, C.S., Jacobsen, T., Marshall, R., Noireaux, V., and Beisel, C.L. (2018). A detailed cellfree transcription-translation-based assay to decipher CRISPR protospacer-adjacent motifs. Methods (in press).

Mohanraju, P., Makarova, K.S., Zetsche, B., Zhang, F., Koonin, E.V., and van der Oost, J. (2016). Diverse evolutionary roots and mechanistic variations of the CRISPR-Cas systems. Science 353, aad5147.

Najm, F.J., Strand, C., Donovan, K.F., Hegde, M., Sanson, K.R., Vaimberg, E.W., Sullender, M.E., Hartenian, E., Kalani, Z., Fusi, N., et al. (2018). Orthologous CRISPR-Cas9 enzymes for combinatorial genetic screens. Nat. Biotechnol. 36, 179-189.

Ng, D.T.W., and Sarkar, C.A. (2012). Model-guided ligation strategy for optimal assembly of DNA libraries. Protein Eng. Des. Sel. 25, 669-678.

Nissim, L., Perli, S.D., Fridkin, A., Perez-Pinera, P., and Lu, T.K. (2014). Multiplexed and programmable regulation of gene networks with an integrated RNA and CRISPR/Cas toolkit in human cells. Mol. Cell 54, 698-710.

Noble, C., Olejarz, J., Esvelt, K.M., Church, G.M., and Nowak, M.A. (2017). Evolutionary dynamics of CRISPR gene drives. Sci Adv 3, e1601964.

Perez-Pinera, P., Kocak, D.D., Vockley, C.M., Adler, A.F., Kabadi, A.M., Polstein, L.R., Thakore, P.I., Glass, K.A., Ousterout, D.G., Leong, K.W., et al. (2013). RNA-guided gene activation by CRISPR-Cas9-based transcription factors. Nat. Methods 10, 973-976.

Peters, J.M., Colavin, A., Shi, H., Czarny, T.L., Larson, M.H., Wong, S., Hawkins, J.S., Lu, C.H.S., Koo, B.-M., Marta, E., et al. (2016). A comprehensive, CRISPR-based functional analysis of essential genes in bacteria. Cell 165, 1493-1506.

Plagens, A., Tripp, V., Daume, M., Sharma, K., Klingl, A., Hrle, A., Conti, E., Urlaub, H., and Randau, L. (2014). In vitro assembly and activity of an archaeal CRISPR-Cas type I-A Cascade interference complex. Nucleic Acids Res. 42, 5125-5138.

Shalem, O., Sanjana, N.E., and Zhang, F. (2015). High-throughput functional genomics using CRISPR-Cas9. Nat. Rev. Genet. 16, 299-311.

Shmakov, S., Abudayyeh, O.O., Makarova, K.S., Wolf, Y.I., Gootenberg, J.S., Semenova, E., Minakhin, L., Joung, J., Konermann, S., Severinov, K., et al. (2015). Discovery and functional characterization of diverse Class 2 CRISPR-Cas systems. Mol. Cell 60, 385-397.

Slaymaker, I.M., Gao, L., Zetsche, B., Scott, D.A., Yan, W.X., and Zhang, F. (2016). Rationally engineered Cas9 nucleases with improved specificity. Science 351, 84-88. 
Swarts, D.C., van der Oost, J., and Jinek, M. (2017). Structural basis for guide RNA processing and seed-dependent DNA targeting by CRISPR-Cas12a. Mol. Cell 66, 221-233.e4.

Szczelkun, M.D., Tikhomirova, M.S., Sinkunas, T., Gasiunas, G., Karvelis, T., Pschera, P., Siksnys, V., and Seidel, R. (2014). Direct observation of R-loop formation by single RNA-guided Cas9 and Cascade effector complexes. Proc. Natl. Acad. Sci. U. S. A. 111, 9798-9803.

Tak, Y.E., Esther Tak, Y., Kleinstiver, B.P., Nuñez, J.K., Hsu, J.Y., Horng, J.E., Gong, J., Weissman, J.S., and Keith Joung, J. (2017). Inducible and multiplex gene regulation using 8 CRISPR-Cpf1-based transcription factors. Nat. Methods 14, 1163-1166.

Tang, X., Lowder, L.G., Zhang, T., Malzahn, A.A., Zheng, X., Voytas, D.F., Zhong, Z., Chen, Y., Ren, Q., Li, Q., et al. (2017). A CRISPR-Cpf1 system for efficient genome editing and transcriptional repression in plants. Nat Plants 3, 17103.

Tsai, S.Q., and Keith Joung, J. (2016). Defining and improving the genome-wide specificities of CRISPR-Cas9 nucleases. Nat. Rev. Genet. 17, 300-312.

Tsai, S.Q., Wyvekens, N., Khayter, C., Foden, J.A., Thapar, V., Reyon, D., Goodwin, M.J., Aryee, M.J., and Joung, J.K. (2014). Dimeric CRISPR RNA-guided FokI nucleases for highly specific genome editing. Nat. Biotechnol. 32, 569-576.

Vercoe, R.B., Chang, J.T., Dy, R.L., Taylor, C., Gristwood, T., Clulow, J.S., Richter, C., Przybilski, R., Pitman, A.R., and Fineran, P.C. (2013). Cytotoxic chromosomal targeting by CRISPR/Cas systems can reshape bacterial genomes and expel or remodel pathogenicity islands. PLoS Genet. 9, e1003454.

Wong, A.S.L., Choi, G.C.G., Cui, C.H., Pregernig, G., Milani, P., Adam, M., Perli, S.D., Kazer, S.W., Gaillard, A., Hermann, M., et al. (2016). Multiplexed barcoded CRISPR-Cas9 screening enabled by CombiGEM. Proc. Natl. Acad. Sci. U. S. A. 113, 2544-2549.

Xie, K., Minkenberg, B., and Yang, Y. (2015). Boosting CRISPR/Cas9 multiplex editing capability with the endogenous tRNA-processing system. Proc. Natl. Acad. Sci. U. S. A. 112, 3570-3575.

Yamano, T., Zetsche, B., Ishitani, R., Zhang, F., Nishimasu, H., and Nureki, O. (2017). Cell 67, 633-645.e3.

Yin, H., Song, C.-Q., Suresh, S., Kwan, S.-Y., Wu, Q., Walsh, S., Ding, J., Bogorad, R.L., Zhu, L.J., Wolfe, S.A., et al. (2018). Partial DNA-guided Cas9 enables genome editing with reduced off-target activity. Nat. Chem. Biol. 14, 311-316.

Zaslaver, A., Bren, A., Ronen, M., Itzkovitz, S., Kikoin, I., Shavit, S., Liebermeister, W., Surette, M.G., and Alon, U. (2006). A comprehensive library of fluorescent transcriptional reporters for Escherichia coli. Nat. Methods 3, 623-628. Essletzbichler, P., Volz, S.E., Joung, J., van der Oost, J., Regev, A., et al. (2015). Cpf1 is a 
1 Zetsche, B., Heidenreich, M., Mohanraju, P., Fedorova, I., Kneppers, J., DeGennaro, E.M.,

2 Winblad, N., Choudhury, S.R., Abudayyeh, O.O., Gootenberg, J.S., et al. (2016). Multiplex gene

3 editing by CRISPR-Cpf1 using a single crRNA array. Nat. Biotechnol. 35, 31-34.

4 Zhong, G., Wang, H., Li, Y., Tran, M.H., and Farzan, M. (2017). Cpf1 proteins excise CRISPR

5 RNAs from mRNA transcripts in mammalian cells. Nat. Chem. Biol. 13, 839-841. 


\section{STARTMETHODS}

\section{KEY RESOURCES TABLE}

\begin{tabular}{|c|c|c|}
\hline REAGENT or RESOURCE & SOURCE & IDENTIFIER \\
\hline \multicolumn{3}{|l|}{ Bacterial strains } \\
\hline E. coli BW25113 $\Delta$ CRISPR $\Delta$ LacOperon & Table S2 & Table S2 \\
\hline S. cerevisiae strain YPH500 & Table S2 & Table S2 \\
\hline E. coli TOP10 & Table S2 & Table S2 \\
\hline E. coli DH5a & Table S2 & Table S2 \\
\hline E. coli Novablue & Table S2 & Table S2 \\
\hline E.coli Tg1 & Table S2 & Table S2 \\
\hline \multicolumn{3}{|l|}{ Chemicals, Peptides, and Recombinant Proteins } \\
\hline Q5 DNA Hot Start High-Fidelity DNA Polymerase & New England Biolabs & M0493 \\
\hline BsmBI & New England Biolabs & R0580S \\
\hline Bsal-HF & New England Biolabs & R3535S \\
\hline \multicolumn{3}{|l|}{ Critical Commercial Assays } \\
\hline myTXTL & Arbor Biosciences & 507024 \\
\hline ZymoPURE Plasmid Midi Prep Kit & Zymo Research & D4200 \\
\hline Gibson Assembly Cloning Kit & New England Biolabs & E5510S \\
\hline NEBuilder HiFi DNA Assembly Cloning Kit & New England Biolabs & E5520S \\
\hline Q5 Site-Directed Mutagenesis Kit & New England Biolabs & E0554S \\
\hline Direct-zol RNA MiniPrep Plus w/ TRI Reagent & Zymo Research & R2071 \\
\hline TURBO DNA-free Kit & Invitrogen & AM1907 \\
\hline T4 Polynucleotide Kinase & New England Biolabs & M0201S \\
\hline RNA Clean and Concentrator Kit & Zymo Research & $\mathrm{R} 1015$ \\
\hline Ribo-Zero rRNA Removal Kit (Bacteria) & Illumina & MRZMB126 \\
\hline $\begin{array}{l}\text { NEBNext Multiplex Small RNA Library Prep Set for } \\
\text { Illumina (Set 1) }\end{array}$ & New England Biolabs & E7300S \\
\hline \multicolumn{3}{|l|}{ Deposited Data } \\
\hline RNA-seq analysis of processed FnCas12a arrays & Table S2 & Table S2 \\
\hline
\end{tabular}




\begin{tabular}{|l|l|l|}
\hline Oligonucleotides & & \\
\hline Primers and cloning oligonucleotides & Table S3 & Table S3 \\
\hline Recombinant DNA & & \\
\hline Plasmids & Table S2 & Table S2 \\
\hline Software and Algorithms & & \\
\hline Geneious 10.2.3 & Geneious & N/A \\
\hline Other & & \\
\hline Detailed protocol for array assembly & Methods S1 & Methods S1 \\
\hline
\end{tabular}

2 CONTACT FOR REAGENT AND RESOURCE SHARING

3 Further information and requests should be directed to the Lead Contact, Chase Beisel

4 (chase.beisel@helmholtz-hiri.de).

\section{METHOD DETAILS}

7 Strains, Plasmids, and Growth Conditions. All strains, plasmids, and oligonucleotides are

8 listed in Table S2 and Table S3. For experiments in E. coli and TXTL, SpCas9 was expressed

9 from pCas9 (Addgene \#42876) while the other cas genes were expressed under the control of

10 the J23108 promoter in a pBAD33 backbone. The targeted plasmids used with the plasmid

11 clearance assays for FnCas12a were constructed by Q5 mutagenesis (New England Biolabs)

12 following the manufacturer's instructions to remove portions of the pUA66-lacZ plasmid (GFP

13 gene driven by lacZ promoter) so that each targeted plasmid only contain one protospacer

14 matching to the designed spacers. The targeted plasmids used with the plasmid clearance

15 assays for SpCas9 were constructed by inserting the fragments containing the PAM and

16 protospacer in between the Xhol and BamHI sites of pUA66. The GFP reporter plasmids used

17 with the gene repression assays were constructed by inserting a constitutive (lac/Q) or inducible

18 promoter (lacZ or $a r a B$ ) into the $\mathrm{Xhol}$ and BamHI restriction sites upstream of the gfp gene in 
1 pUA66 (Zaslaver et al., 2006). The reporter plasmid for in vitro RNA detection with LsCas13a

2 was the p70a-deGFP plasmid reported previously (Garamella et al., 2016). The target encoding

3 plasmids were constructed by inserting the protospacer into the plasmid pUA66_PJ23119 by

4 replacing the ORF of GFP gene. Plasmid p70a-deGFP was also used for the off-target binding

5 experiments by inserting each target sequence to upstream of the P70a promoter using Q5

6 mutagenesis. The Cas $12 \mathrm{a}$ encoding plasmids were constructed by inserting the open reading

7 frame of FnCpf1 or AsCpf1 into pBAD33 with a constitutive promoter (PJ23108). The plasmids

8 encoding $\mathrm{dFnCas} 12 \mathrm{a}$ variants were constructed by Q5 mutagenesis using the wild type

9 FnCas12a plasmid as template. Plasmid used for expressing Cas9 was pCas9 (Addgene \#

10 42876). Plasmid pCas9-notracr was constructed by removing the tracrRNA portion from the

11 plasmid pCas9 using Q5 mutagenesis. The in vivo plasmid clearance assays were conducted in

12 CB414, a derivative of E. coli BW25113 with the lacl promoter through the lacZ gene and the 13 endogenous I-E CRISPR-Cas system deleted.

14 E. coli cells were grown in Luria Bertani (LB) medium (10 g/L NaCl, $5 \mathrm{~g} / \mathrm{L}$ yeast extract,

$1510 \mathrm{~g} / \mathrm{L}$ tryptone) at $37^{\circ} \mathrm{C}$ with shaking at $250 \mathrm{rpm}$. The antibiotics ampicillin, chloramphenicol, 16 and/or kanamycin were added to maintain any plasmids at $50 \mu \mathrm{g} / \mathrm{mL}, 34 \mu \mathrm{g} / \mathrm{mL}$, and $50 \mu \mathrm{g} / \mathrm{mL}$, 17 respectively. The inducers Isopropyl $\beta$-D-1-thiogalactopyranoside (IPTG) or L-arabinose were 18 added at concentrations of $0.2 \mathrm{mM}$ and $0.2 \%$ when specified.

The S. cerevisiae strain YPH500 (Stratagene) was used as the background strain for 20 gene activation. Culturing and genetic transformation were done as previously described (Khalil 21 et al., 2012) using either URA3, HIS3, or LEU2 as selectable markers. The reporter plasmid for 22 gene activation was constructed from integrative plasmid pRS406 (Strata-gene) as described 23 previously (Keung et al., 2014). A CEN pRS414 plasmid was used to express the FnCas12a 24 variants from a GPD promoter. To construct FnCas12a-VP64, the FnCas12a double mutant 25 was cloned in between pGPD and VP64 in pRS414 through Gibson assembly. All plasmid 
constructs were generated using TOP10, Novablue, Tg1, or DH5a electrocompetent cells and verified by PCR and Sanger Sequencing of the inserted sequence.

4 Generation of CRISPR arrays. The backbone plasmid used for generating CRISPR arrays for

5 FnCas12a (pFnCpf1GG) was constructed by Gibson assembly to join three PCR fragments

6 together and kill the extra BsmBI site on the scaffold backbone. The three PCR fragments

7 contain a constitutive J23119 promoter, a GFP dropout construct (with promoter and terminator)

8 flanked by two Type IIS BsmBI restriction sites and a direct repeat of FnCas12a, and rrnB

9 terminator, ampicillin resistance gene, and pMB1 origin of replication, respectively. The

10 backbone plasmid used for generating arrays for AsCas12a, and LsCas13a, and the 4-spacer

11 composite arrays were constructed using Q5 mutagenesis to remove the FnCas12a repeat and

12 insert the other corresponding repeats. The backbone plasmid used for generating arrays for

13 SpCas9 was constructed by inserting a PCR fragment with the Cas9 direct repeat and BsmBI

14 sites flanked mRFP dropout construct into Aatll and Hindlll digested pFnCpf1GG. Backbone

15 plasmid for generating composite arrays for blocking off-target cleavage was constructed using

16 Q5 mutagenesis to remove extra nucleotides on the 3' of the promoter from pFnCpf1GG, so that

17 the transcription starts from the sgRNA of Cas9. Backbone plasmid for generating arrays to use

18 in yeast was constructed by using Gibson Assembly to join three PCR fragments and mutate a

19 Bsal site on the scaffold backbone: Bsal sites flanked GFP dropout construct and two PCR

20 fragments amplified from plasmid pRS413, on which arrays are transcribed from a SNR52

21 snoRNA promoter. The sequences of the resulting backbone plasmids are available in Table

22 S2. Forward and reverse oligonucleotides encoding one repeat, one spacer, and a 4-nt junction

23 were annealed to form dsDNA with a 5' and/or 3' overhangs. $400 \mathrm{fmol}$ of each dsDNA, $20 \mathrm{fmol}$

24 of backbone plasmid, $1 \mu \mathrm{l}$ of T7 ligase, and $1 \mu \mathrm{l}$ of BsmBI or Bsal were added to $2 \mu \mathrm{l}$ of T4

25 ligation buffer, then water was added to reach a total volume of $20 \mu \mathrm{l}$. A thermocycler was used 
1 to perform 25 cycles of digestion and ligation $\left(42{ }^{\circ} \mathrm{C}\right.$ for $2 \mathrm{~min}, 16^{\circ} \mathrm{C}$ for $5 \mathrm{~min}$ ) followed by a

2 final digestion step $\left(60^{\circ} \mathrm{C}\right.$ for $\left.10 \mathrm{~min}\right)$, and a heat inactivation step $\left(80^{\circ} \mathrm{C}\right.$ for $\left.10 \mathrm{~min}\right)$. The ligation

3 mix was then diluted 1:6 in water and electroporated into competent $E$. coli cells. After

4 transformation and recovery for 1 hour at $37^{\circ} \mathrm{C}$ with shaking at $250 \mathrm{rpm}$ in SOC media, cells

5 were plated on LB agar containing the appropriate antibiotic and incubated for $16 \mathrm{~h}$. White

6 colonies were then screened for the presence of the correct band size, and the array was

7 validated through Sanger sequencing of the PCR product. See Methods S1 for a detailed

8 protocol and an example of assembling one of the three-spacer FnCas12a arrays.

9 The no-spacer control used in most of the experiments was generated by inserting a 10 single repeat into the backbone plasmid, resulting in two consecutive repeats with no 11 intervening spacer.

12 Composite arrays for coordinated plasmid clearance and gene repression were

13 generated using the same method except that the backbone plasmid had a 5' direct repeat for

14 SpCas9 and and 3' direct repeat for FnCas12a. Composite arrays for reducing off-targeting 15 were generated by assembling the Cas9 sgRNA and two repeat-spacer dsDNAs into the GFP 16 drop-out backbone. The sgRNA was formed with two annealed oligonucleotides, in line with 17 CRATES.

19 In vivo plasmid clearance assay. We transformed 50 ng of the plasmid encoding the CRISPR 20 array or a non-targeting control with no spacer into $E$. coli cells harboring a plasmid encoding a 21 Cas protein and another plasmid encoding the gRNA target sequence. After recovering for one 22 hour in SOC at $37^{\circ} \mathrm{C}$ with shaking at $250 \mathrm{rpm}$, cells were serially diluted and $10 \mu \mathrm{l}$ of droplet was 23 plated on LB agar plates with ampicillin, kanamycin, and chloramphenicol. After 16 hours of 24 growth, colony numbers were recorded for analysis. 
In vivo gene repression assay. CB414 cells were initially transformed with three compatible plasmids: a plasmid encoding a variant of FnCas12a, a plasmid encoding a CRISPR array or no-spacer control, and a plasmid encoding GFP under the control of the $l a c Z$, laclQ, or araB

4 promoter. Overnight cultures of cells harboring the three plasmids were back-diluted to $\mathrm{ABS}_{600}$ $5 \sim 0.01$ in LB medium with ampicillin, kanamycin and chloramphenicol and the promoter's inducer 6 and shaken at $250 \mathrm{rpm}$ at $37^{\circ} \mathrm{C}$ until $\mathrm{ABS}_{600}$ reach $\sim 0.2$. Cultures were then diluted 1:25 in $1 \mathrm{X}$ 7 phosphate buffered saline (PBS) and analyzed on an Accuri C6 flow cytometer with C6 sampler

8 plate loader (Becton Dickinson) equipped with CFlow plate sampler, a 488-nm laser, and a 530

$9+/-15-n m$ bandpass filter. GFP fluorescence was measured as described previously (Leenay et 10 al., 2016). Briefly, forward scatter (cut-off of 15,500) and side scatter (cut-off of 600) were used

11 to eliminate non-cellular events. The mean value within FL1-H of 30,0000 events within a gate

12 set for E. coli (Afroz et al., 2014) were used for data analysis.

14 Small-RNA library preparation, sequencing, and data analysis. Plasmids encoding 15 FnCas12a and the arrays were added into 9 ul of MyTXTL master mix (Arbor Biosciences) to a 16 final concentration of $5 \mathrm{nM}$ each in a PCR tube and a total volume of 12 ul. Two uM of Chi6 17 annealed oligos was included in the reaction to prevent any unintentional degradation of the 18 plasmids by RecBCD proteins. The mixture was incubated at $29^{\circ} \mathrm{C}$ for five hours in a 19 thermocycler, and total RNA was extracted using Direct-zol RNA MiniPrep kit following the 20 manufacturer's instructions (Zymo Research). After DNase treatment with Turbo DNase (life 21 Technologies), and 3' dephosphorylation with T4 Polynucleotide Kinase (New England Biolabs), 22 rRNAs were then depleted with the Ribo-Zero Bacteria Kit (Illumina) following the 23 manufacturer's instructions. RNA libraries were prepared using NEBNext Multiplex Small RNA 24 Library Prep Set for Illumina (New England Biolabs) following the manufacturer's instructions. 25 Samples were sequenced on a MiSeq machine (Illumina) by the Genomic Science Laboratory 
1 at North Carolina State University. The Geneious 10.2.3 software package (Biomatters) was

2 used for data sorting and alignment. Briefly, Fastq reads were trimmed and quality filtered using

3 the BBDuk plugin. Trimmed reads were then aligned to created CRISPR array reference

4 sequences (Fncpf1_7_spacer_array, cF-2/3/1, or cF-1/3/2) using Geneious 10.2.3' Map to

5 Reference, high sensitivity setting.

6

7 In vitro multiplexed RNA detection with Cas13a. Open reading frame of LsCas13a was

8 cloned into pBAD33 with a constitutive promoter. Single or multiple spacer arrays were

9 generated as described. Targeted RNAs were transcribed using a constitutive promoter on the

10 plasmid. A plasmid constitutively express a deGFP protein was used as reporter. The Cas $13 a$

11 encoding plasmid, array encoding plasmid, and target encoding plasmid were added into 9 ul of

12 MyTXTL master mix (Arbor Biosciences) to a final concentration of $2 \mathrm{nM}, 1 \mathrm{nM}$ and $0.5 \mathrm{nM}$

13 respectively, to a total volume of $12 \mathrm{ul}$, and incubated at $37^{\circ} \mathrm{C}$ for 2 hours. Then the reporter

14 plasmid was added to a final concentration of $0.5 \mathrm{nM}$. Aliquots of $5 \mathrm{ul}$ were placed to the wells of

15 96-well V-bottom plate (Corning Costar 3357) and incubated at $37^{\circ} \mathrm{C}$ for 16 hours in a Synergy

16 H1MF microplate reader (BioTek) with kinetic reading every 3 minutes (excitation, emission: 485

17 nm, 528 nm; gain: 60; lightsource: Xenon Flash).

19 Gene activation in Saccharomyces cerevisiae. Three single yeast colonies for each strain

20 were picked after plasmid transformations and inoculated into $500 \mathrm{ml}$ of SD-media (synthetic

21 dropout media containing $2 \%$ glucose with defined amino acid mixtures) in Costar 96-well assay

22 blocks (V-bottom; $2 \mathrm{ml}$ max volume; Fisher Scientific). The cultures were grown at $30{ }^{\circ} \mathrm{C}$ with

$23250 \mathrm{rpm}$ shaking for $24-48 \mathrm{hr}$. Cultures were then re-inoculated in SD-complete media to an

$24 \mathrm{OD} 600$ of 0.05 to 0.1 and grown at $30{ }^{\circ} \mathrm{C}$ with $250 \mathrm{rpm}$ shaking for 12 hours. Cells were treated

25 with $10 \mathrm{mg} / \mathrm{ml}$ cycloheximide to inhibit protein synthesis and then assayed for $y E G F P$ 
1 expression by flow cytometry. 10,000 events were acquired using a MACSQuant VYB flow

2 cytometer with 96-well plate sampler. Events were gated by forward scatter and side scatter and

3 all values obtained were from three isogenic strains. Plots were generated based on side scatter

4 versus FL1 fluorescence.

5

6 In vitro assessment of blocking off-target cleavage. Single guides for Cas 9 cleavage, single

7 spacer arrays for dFnCpf1 blocking off-target sites, and composite arrays for cleavage and

8 blocking synchronously were generated as described. The protospacers were cloned into

9 upstream of constitutive promoter of GFP gene on a plasmid. Plasmid encoding dFnCpf1,

10 targeting plasmid for Cas9, targeting plasmid for dFnCpf1 or non-targeting control, and targeted

11 plasmid were added into MyTXTL master mix to final concentration of $1 \mathrm{nM}, 2 \mathrm{nM}$, and $0.5 \mathrm{nM}$

12 respectively, and incubated at $29^{\circ} \mathrm{C}$ for 4 hours. Then the SpCas9 encoding plasmid (pCas9-

13 notracr) was added to the mixture to a final concentration of $2 \mathrm{nM}$ and incubated at $29^{\circ} \mathrm{C}$ for 16

14 hours in a microplate reader with kinetic reading (excitation, emission: $485 \mathrm{~nm}, 528 \mathrm{~nm}$ ) every 3

15 minutes. Non-targeting control was also tested as a control.

\section{QUANTIFICATION AND STATISTICAL ANALYSIS}

18 Plasmid transformation in E. coli. Fold reduction was calculated as the ratio of colony-forming 19 units (CFU's) for cells transformed with the no-spacer array plasmid over that for cells 20 transformed with the CRISPR array plasmid.

22 Fluorescence measurements in E. coli. GFP fold-repression was calculated using mean

23 fluorescence values, subtracting the fluorescence of $E$. coli cells lacking the GFP reporter 24 plasmid, and dividing the resulting fluorescence value for the no-spacer array by that for the 25 tested CRISPR array. 
2 Fluorescence measurements in yeast. The fraction of GFP-positive cells were calculated by

3 setting a threshold on FL1 so $<0.1 \%$ of the cells lacking a gRNA fall within the the GFP-positive

4 bin. The reported fraction is the percentage of cells that fall above the threshold within the entire

5 gated population.

6

7 RNA sensing in TXTL. End-point fluorescence measurements are reported as a heat map.

8

9 Blocking off-target cleavage in TXTL. The transcriptional rate was calculated as the slope of

10 each fluorescence signal curve from 2.5 hours to 4.5 hours after the pCas9-notracr plasmid was

11 added. Protection percentage was calculated as the ratio of the transcriptional rate for CRISPR

12 array over that of the non-targeting control.

\section{DATA AND SOFTWARE AVAILABILITY}

15 Next-generation sequencing data for the RNA-seq analysis of gRNA abundance will be 16 accessible through NCBI Biosample. 
A

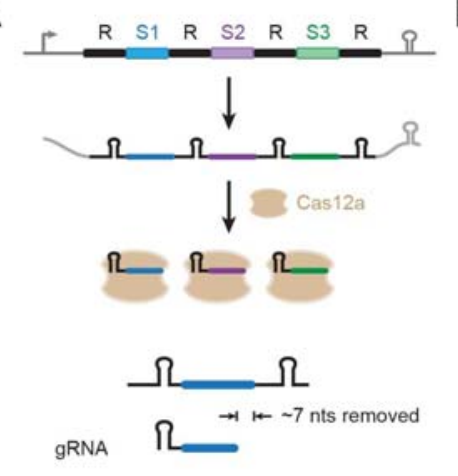

C

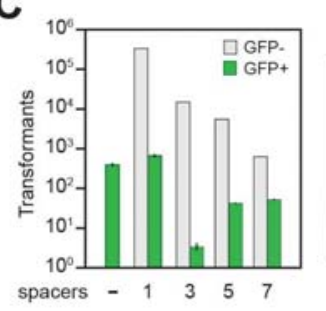

D

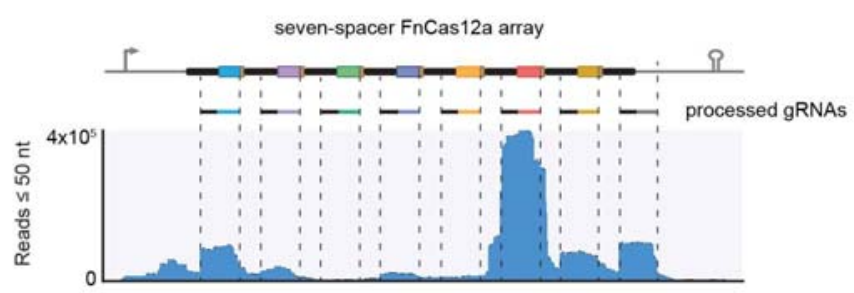

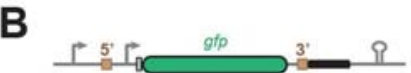

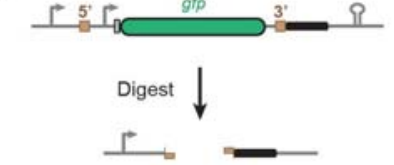

Ligate $\mid \begin{aligned} & \mathrm{A}^{\prime}=\mathrm{A} \\ & \mathrm{A}=\mathrm{B}^{\prime}\end{aligned}$
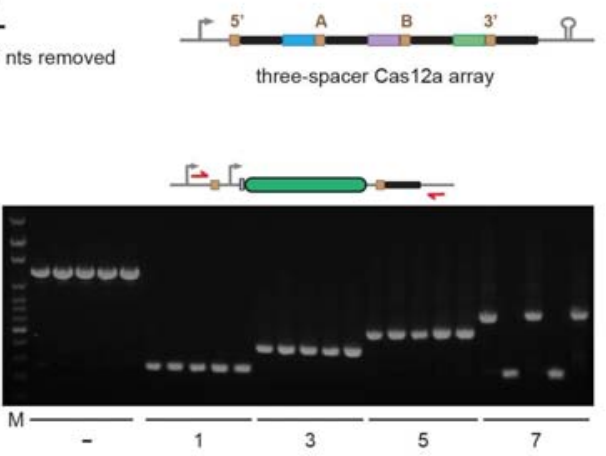

seven-spacer FnCas12a array$$
\text { (1) }
$$

4 Figure 1. A one-step assembly scheme for CRISPR-Cas12a arrays based on spacer trimming.

5 (A) CRISPR array processing by the Cas12a nuclease. Each array comprises alternating

6 repeats ( $\mathrm{R}$, black bars) and spacers (S1-S3, colored bars). Processing includes 3' trimming of

7 the spacer to form the final gRNA. (B) Cloning scheme for the one-step assembly of multi-

8 spacer arrays recognized by Cas12a. A GFP-dropout construct is flanked by two Type IIS

9 restriction sites and a 3' direct repeat. The digested construct is ligated with annealed

10 oligonucleotides encoding individual repeat-spacers in one step. The initial repeat is located at

11 the 3' end so the resulting array begins and ends with a repeat. The sequence and 5' or 3'

12 directionality of the junction overhangs determine the order of assembly. The resulting assembly 
1 junctions fall within the trimmed portion of the processed guide RNA and therefore would not be

2 involved in target recognition. (C) Efficient assembly of up to a seven-spacer array. Assembly

3 efficiency was based on the relative proportion of fluorescent or non-fluorescent $E$. coli colonies

4 (left) and the correct insert size of non-fluorescent colonies subjected to colony PCR (right).

5 Values represent the geometric average and S.E.M. from three independent transformations

6 starting from separate colonies. See Table S3 for the specific sequences used. - is the original

7 GFP dropout construct. (D) RNA-seq analysis of the transcribed 7-spacer array. The assembled

8 array and FnCas12a were expressed in an all-E. coli cell-free transcription-translation reactions.

9 Reads of no more than 50 nts were mapped to the original array expression construct. Related

10 to Figure S1 and Tables S1-S3. 
A

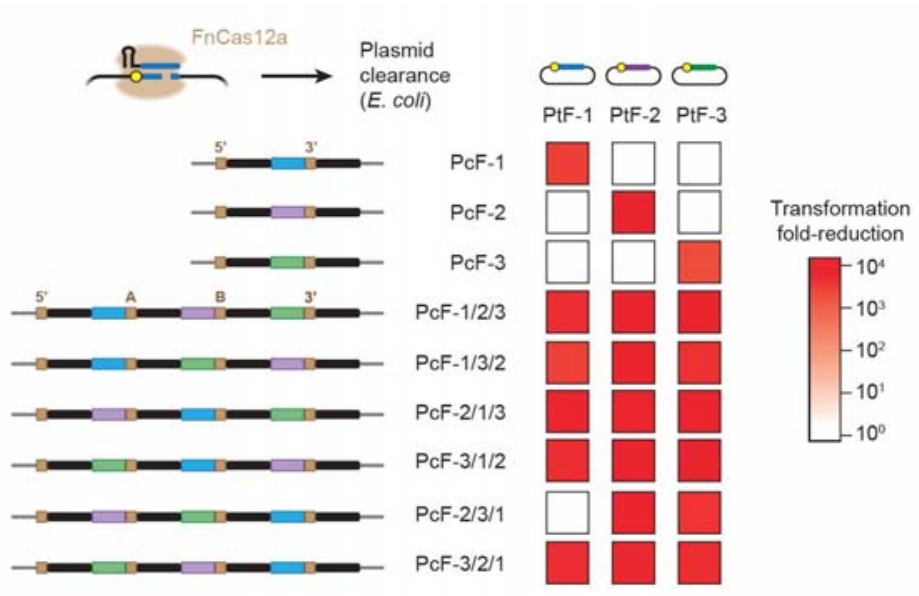

B
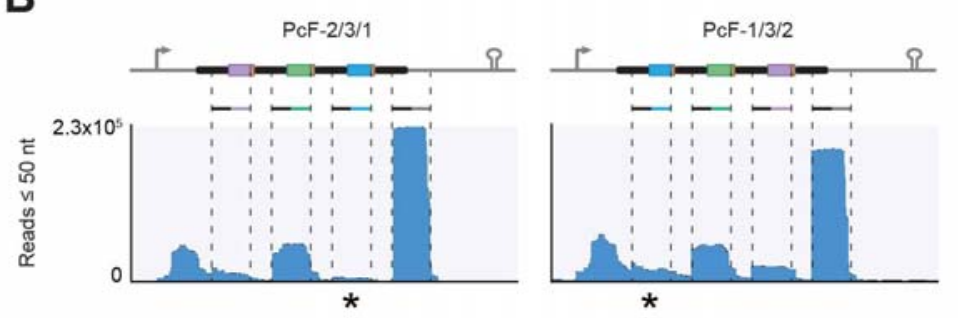

2 Figure 2. Plasmid clearance with assembled CRISPR-Cas12a arrays reveals contextdependent spacer activity. (A) Multiplexed plasmid clearance by FnCas12a in E. coli. Spacers

4 (colored bars) were designed to target a distinct protospacer flanked by a PAM (yellow circle) in

5 a transformed plasmid. E. coli cells harboring the FnCas12a plasmid and the target plasmid were transformed with a plasmid encoding the indicated CRISPR array or a no-spacer array,

7 and the fold-change in the number of transformants is reported as a heat map. Sequences of

8 the spacers and junctions in the assembled arrays are shown in Table S3. Values represent the

9 average of at least three independent transformation experiments starting from separate

10 colonies. (B) RNA-seq analysis of three-spacer arrays with differing activity of spacer S1. The

11 assembled array and FnCas12a were expressed in an all-E. coli cell-free transcription-

12 translation reactions. Reads of no more than $50 \mathrm{nts}$ were mapped to the original array

13 expression construct. Stars indicate mapped reads for spacer 1. Related to Figures S2-S3 and

14 Tables S2-S3. 

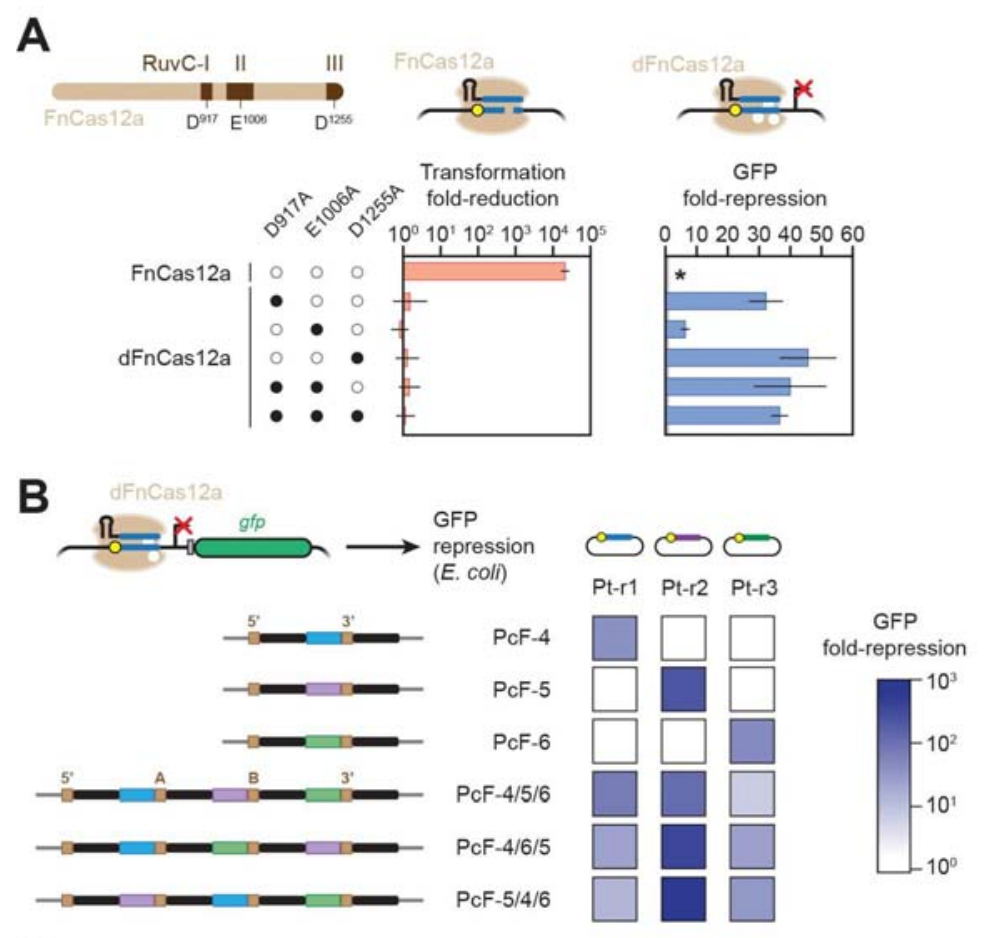

C

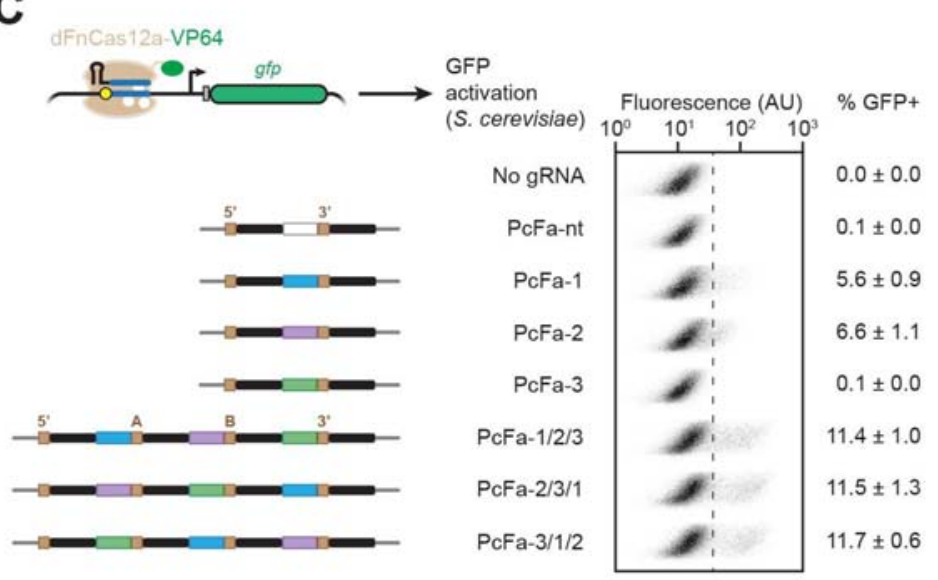

2 Figure 3. Assembled CRISPR-Cas12a arrays allow multiplexed gene regulation in E. coli and

3 yeast. (A) Impact of RuvC mutations on plasmid clearance and gene repression by FnCas12a in

4 E. coli. Transformations were conducted as described in Figure 2A. GFP repression was

5 measured by flow cytometry analysis with cells harboring the dFnCas12a plasmid, a plasmid

6 harboring a targeting or no-spacer array, and the GFP reporter plasmid. Values represent the

7 average and S.E.M. of at least three independent experiments from separate colonies. The WT

8 FnCas12a was not tested in the GFP-repression assay (starred) because of its strong plasmid- 
1 clearance activity. (B) Multiplexed gene repression with FnCas12a in E. coli. See (A) for details,

2 where the D917A, E1006A mutant of FnCas12a was used. Values represent the average and

3 S.E.M. of at least three independent experiments starting from separate colonies. (C)

4 Multiplexed gene activation with a FnCas12a-VP64 fusion in S. cerevisiae. Fluorescence

5 distributions were generated from flow cytometry analysis and plotting fluorescence versus side

6 scatter. Values represent the average and S.E.M. of at least three independent experiments

7 starting from separate colonies. All arrays were assembled using the junctions specified in

8 Table S3. Related to Figure S4 and Tables S2-S3. 

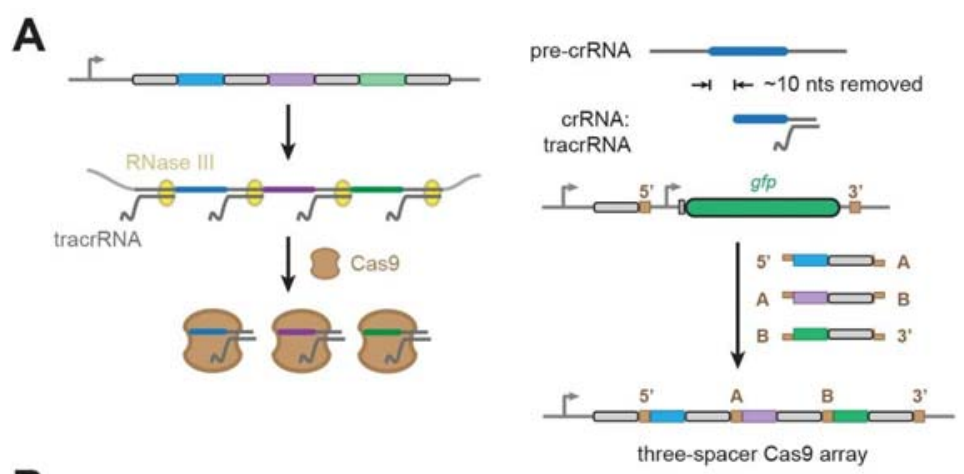

B

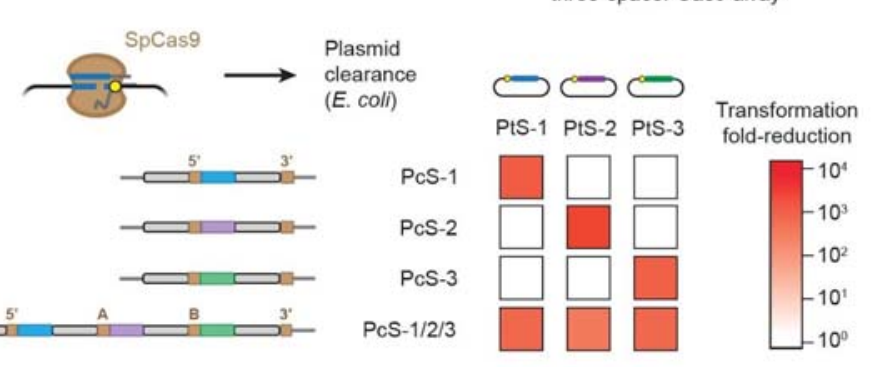

\section{C}
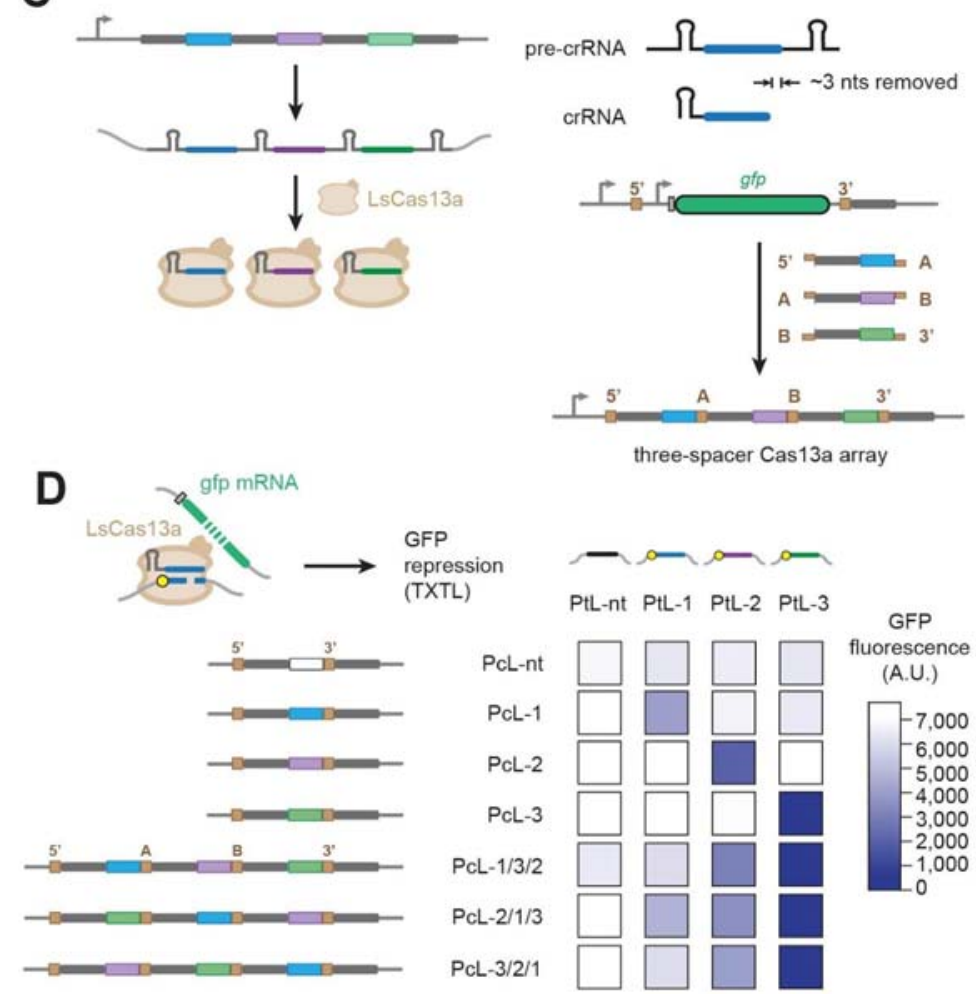

2 Figure 4. Assembled arrays extend to other Class 2 Cas nucleases. (A) Processing mechanism

3 and assembly scheme for CRISPR-Cas9 arrays. The assembly junction is at the 5' end of the

4 spacer to match the location of spacer trimming. (B) Multiplexed plasmid clearance by SpCas9 
1 in E. coli. See Figure 2A for details. Values represent the average of at least three independent

2 experiments starting from separate colonies. (C) Processing mechanism and assembly scheme

3 for CRISPR-Cas13a arrays. The assembly junction is at the 3' end of the spacer to match the

4 location of spacer trimming. (D) Multiplexed RNA sensing by LsCas13a in a cell-free

5 transcription-translation system. Reactions were conducted for $16 \mathrm{~h}$ following the addition of the

6 LsCas13a plasmid, the indicated array plasmid, a plasmid expressing one of the targets, and

7 the GFP plasmid. Target recognition leads to non-specific degradation of the GFP mRNA by

$8 \mathrm{LsCas} 13 \mathrm{a}$, thereby reducing GFP production. End-point fluorescence measurements are

9 reported. Values represent the average of three TXTL experiments and are representative of at

10 least three experiments conducted on different days. All arrays were assembled using the

11 junctions specified in Table S3. See Figure S5 for the assay and GFP timeccourses. Related to

12 Figure S5 and Tables S2-S3. 
A

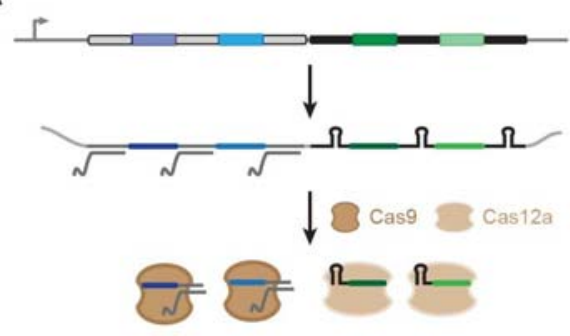

C

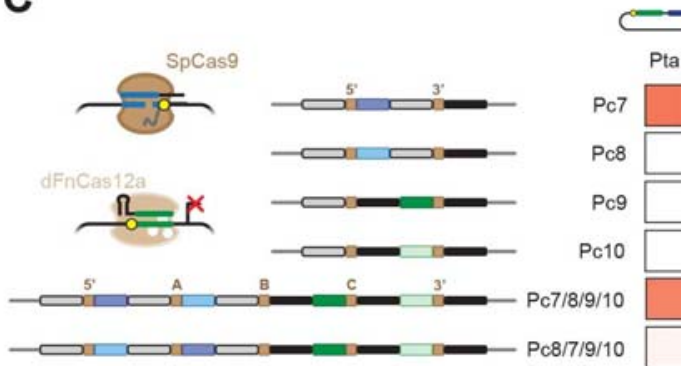

D
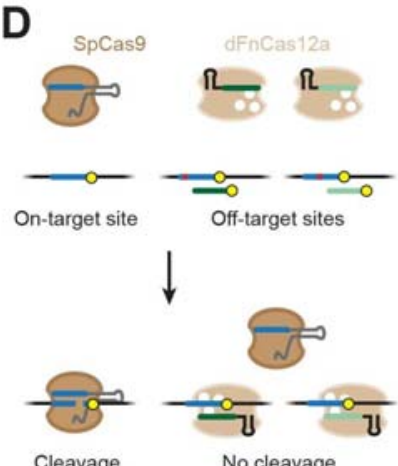

$\mathrm{E}$

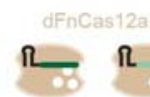

No cleavage
B

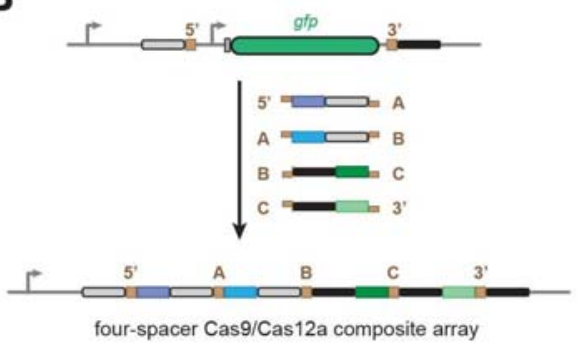

four-spacer Cas $9 /$ Cas 12 a composite array

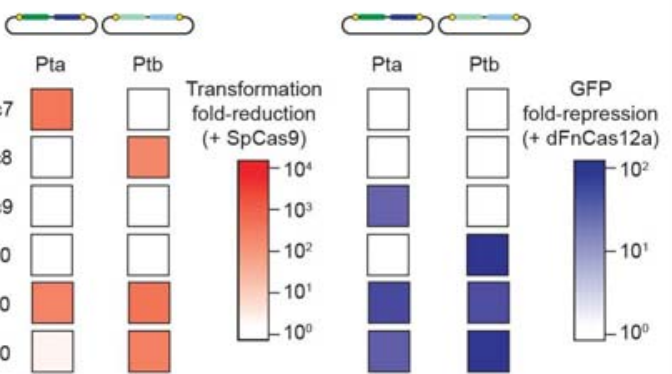

Figure 5. Composite arrays allow recognition and processing by multiple nucleases. (A)

3 Processing of a composite array encoding spacers used by Cas9 and Cas12a. (B) One-step

4 assembly of composite arrays. An array encoding two CRISPR-Cas9 spacers and two CRISPR-

5 Cas12a spacers is shown. The GFP-dropout construct contains an upstream SpCas9 repeat

6 and a downstream FnCas12a repeat to accommodate the orientations of the assembly

7 junctions. (C) Coordinated plasmid clearance by SpCas9 and gene repression by FnCas12a in

8 E. coli. To assess plasmid clearance, cells harboring the SpCas9 plasmid and a target plasmid

9 were transformed with a plasmid encoding the indicated CRISPR array or a no-spacer array. To

10 investigate the GFP repression, cells harboring the dFnCas12a (D917A, E1006A, D1255A)

11 plasmid, a target plasmid, and a plasmid encoding the indicated CRISPR array or a no-spacer 
1 array were assessed by flow cytometry analysis. Values represent the average of at least three

2 independent experiments starting from separate colonies. See Table S2 and S3 for more

3 information on the target constructs. (D) Enhancing the specificity of DNA cleavage by SpCas9

4 by blocking off-target sites with dCas12a. Binding of a known off-target location would block

5 Cas 9 from accessing this site, thereby reducing unintended cleavage at this site. (E) Enhancing

6 DNA cleavage specificity in a model cell-free TXTL system using a composite array composed

7 of one SpCas9 sgRNA and a two-spacer FnCpf1 array. Protection from cleavage was

8 calculated based on the relative rates of GFP production compared to target and non-targeting

9 controls. See methods for details. Values represent the average of at least three independent

10 TXTL experiments conducted on separate days. See Figure S6 for details of the target sites and

11 a representative set of GFP time-course measurements. Related to Figure S6 and Tables S2-

12 S3. 